\title{
脱水糖苷化方法合成肝素酶底物寡糖
}

\author{
许伟长 ${ }^{a}$ 刘威 ${ }^{b}$ 李祥 ${ }^{c}$ 徐鹏*,a 俞风䢠*,a,b \\ ( ${ }^{a}$ 中国科学院上海有机化学研究所 生命有机化学国家重点实验室 上海 200032) \\ ${ }^{b}$ 上海科技大学 物质科学与技术学院 上海 201210) \\ ( ${ }^{c}$ 香港大学 化学系 香港 999077)
}

\begin{abstract}
摘要 乙酰肝素酶(Heparanase, Hpa)是哺乳动物体内的内切葡萄糖醛酸苷水解酶, 通过水解葡萄糖醛酸 $(\mathrm{GlcA})$ 与己胺 糖 $(\mathrm{GlcN})$ 之间的 $\beta$-糖苷键, 选择性地降解肝素和硫酸肝素糖胺聚糖, 从而释放多功能的肝素寡糖. 本文报道一条高效 的肝素酶底物寡糖的合成路线: 采用苯甲酰基保护待硫酸化的羟基，苄基保护羧基和裸露的羟基，叠氮基保护氨基， 应用脱水糖苷化方法高效地构建关键的 $\alpha-\mathrm{GlcN}-(1 \rightarrow 4)-\mathrm{GlcA}$ 糖苷键. 然后通过标准化的保护基脱除和硫酸化操作，获 得肝素酶底物三糖和四糖 1-4. 最后五步反应的总收率超过 $52 \%$. 肝素酶底物寡糖的合成为研究肝素酶的底物选择性 和活性检测打下了基础.
\end{abstract}

关键词 乙酰肝素酶; 肝素葟糖; $\alpha-(1 \rightarrow 4)$ 糖苷键; 脱水糖苷化; 合成

\section{Synthesis of Oligosaccharides Relevant to the Substrates of Heparanase via Dehydrative Glycosylation}

\author{
$\mathrm{Xu}$, Weichang $^{a} \quad$ Liu, Wei $^{b} \quad \mathrm{Li}, \mathrm{Xiang}^{c} \quad \mathrm{Xu}$, Peng $^{*, a} \quad \mathrm{Yu}, \mathrm{Biao}^{*, a, b}$ \\ ( ${ }^{a}$ State Key Laboratory of Bioorganic and Natural Products Chemistry, Shanghai Institute of Organic Chemistry, \\ Chinese Academy of Sciences, Shanghai 200032, China) \\ ( ${ }^{b}$ School of Physical Science and Technology, ShanghaiTech University, Shanghai 201210) \\ ( ${ }^{c}$ Department of Chemistry, The University of Hong Kong, Hong Kong 999077)
}

\begin{abstract}
Heparanase, an endo- $\beta$-D-glucuronidase responsible for specific cleavage of heparin and heparan sulfates, is relevant to a number of biological processes, such as inflammation, tumor angiogenesis and metastasis. Heparin and heparan sulfate(HS), ubiquitously distributed on the cell surface and in the extracellular matrix, play significant roles in a diverse set of biological processes, including cell growth, virus infection, and tumor metastasis. The substrate specificity of the purified recombinant human heparinase has been investigated, and an optimal tetrasaccharide substrate of heparinase was found to be $\Delta \mathrm{HexUA}(2 \mathrm{~S})-\mathrm{GlcN}(\mathrm{NS}, 6 \mathrm{~S})-\mathrm{GlcUA}-\mathrm{GlcN}(\mathrm{NS}, 6 \mathrm{~S})$. Here we report an efficient alternative to the chemical synthesis of oligosaccharides relevant to the substrates of heparanase, including the stereoselective construction of $\alpha$-GlcN-(1 $\rightarrow 4)$-GlcA glycoside bonds and the effective post-assembly manipulations on the fully elaborated oligosaccharides. The dehydrative glycosylation protocol, capitalizing on direct activation of $\mathrm{C} 1$-hemiacetals as glycosyl donors, was employed to construct the challenging $\alpha$-GlcN-(1 $\rightarrow 4)$-GlcA linkages, using diphenyl sulfoxide $\left(\mathrm{Ph}_{2} \mathrm{SO}\right) /$ triflic anhydride $\left(\mathrm{Tf}_{2} \mathrm{O}\right)$ as promoters, 2,4,6-tri-tertbutylpyrimidine(TTBP) as base, toluene as a solvent, and $-60{ }^{\circ} \mathrm{C}$ to room temperature as the working temperature. Under these optimized conditions, mono- and disaccharide donors $(\mathbf{9}$ and $\mathbf{1 0})$ and disaccharide acceptors $(\mathbf{1 1}$ and $\mathbf{1 2})$ were condensed to provide the coupled tri- and tetrasaccharides $\mathbf{5} \sim \mathbf{8}$ in good yields and satisfactory stereoselectivity $(>65 \%$ yield and $\alpha / \beta>$ 5.4/1.0). The fully elaborated oligosaccharides $5 \sim 8$ have then been successfully transformed into the target heparin oligosaccharides $\mathbf{1} \sim \mathbf{4}$ via an effective sequence of manipulation of the protecting groups( $>52 \%$ yield for 5 steps). The post-assembly manipulations include saponification under Zemplén conditions(for removal of benzyl ester and benzoyl group), $\mathrm{O}$-sulfonation with sulfur trioxide pyridine complex(for hydroxyl groups), reduction and $\mathrm{N}$-sulfonation(for azido group), and high pressure hydrogenation(for removal of benzyl groups). The availability of these heparin oligosaccharides would facilitate in-depth elucidation of the substrate selectivity of heparanase and the development of an effective assay for measuring the heparanase activities.
\end{abstract}

Keywords heparanase; heparin oligosaccharide; $\alpha-(1 \rightarrow 4)$ glycosidic bond; dehydrative glycosylation; chemical synthesis

\footnotetext{
* E-mail: peterxu@sioc.ac.cn; byu@sioc.ac.cn; Tel.: 021-54925131

Received June 1, 2020; published June 28, 2020.

Supporting information for this article is available free of charge via the Internet at http://sioc-journal.cn.

Financial support from National Natural Science Foundation of China (Nos. 21621002 \& 21602240), Key Research Program of Frontier Sciences of CAS (No. ZDBS-LY-SLH030), Strategic Priority Research Program of CAS (No. XDB20020000), and Youth Innovation Promotion Association of CAS (No. 2020258) are acknowledged.

本项目受国家自然科学基金委员会(Nos. 21621002 \& 21602240), 中科院前沿科学重点研究项目(No. ZDBS-LY-SLH030), 中国科学院战略性先导科 技专项(B 类)(No. XDB20020000)和中科院青年创新促进会(No. 2020258)的资助.
} 


\section{1 引言}

肝素(Heparin)和硫酸肝素(Heparan Sulfate)是一类 高度硫酸化的天然直链聚糖, 由重复的二糖单元: $1 \rightarrow 4$ 连接的己糖醛酸 ( $\alpha$ - $\mathrm{L}$-艾杜糖醛酸或者 $\beta$ - $\mathrm{D}$-葡萄糖醛酸) 与己糖胺 $(\alpha-\mathrm{D}$-氨基葡萄糖)交替连接而成, 其分子量分 布在 5000 30000 之间. 肝素和硫酸肝素的硫酸化位点 主要在氨基葡萄糖基的 3 位、6 位和糖醛酸的 2 位, 另 外, 氨基葡萄糖基的 2 位氨基可以有硫酸化、乙酰化和 裸露三种形式.

肝素最早由约翰霍普金斯大学的 McLean 和 Howell 于 1916 年从动物的肝脏中分离得到, 并发现了它具有 强抗凝血作用 ${ }^{[1]}$. 近年来, 受益于生物学技术的发展, 肝素类分子越来越多的生物学功能被揭示 ${ }^{[2]}$. 特别是其 独特的抗凝血作用机理得到阐明 ${ }^{[3]}$ : 肝素主要通过与抗 凝血酶III(ATIII)的结合, 抑制凝血酶原激活物和凝血酶 的形成和活性, 从而促进纤维蛋白(血栓)的溶解; 并且 通过抑制血小板的粘附和聚集, 间接影响血小板内凝血 因子的释放. 除了具有抗凝血和抗血栓的功能外, 肝素 还具有降血脂、抗动脉粥样硬化、抗中膜平滑肌细胞 (SMC)增生、抗炎、抗过敏、抗病毒、抗癌等多种生物 学功能 ${ }^{[4]}$.

由于肝素结构的复杂性和微观不均一性, 从天然肝 素中分离得到结构单一的片断非常困难. 同时, 肝素结 构中众多的 $O$-硫酸基、 $N$-硫酸基以及羧酸基对其生物活 性的影响非常大. 通过合成手段得到精确结构的肝素臭 糖, 对于其构效关系和生物学研究是极其必要的 ${ }^{[3]}$. 确 实, 肝素臭糖的合成一直是备受化学家关注的课题 ${ }^{[2,5]}$.

乙酰肝素酶(Heparanase, Hpa)是哺乳动物体内的内 切葡萄糖醛酸水解酶, 它能通过选择性水解葡萄糖醛酸 $(\mathrm{GlcA})$ 与已胺糖 $(\mathrm{GlcN})$ 之间的 $\beta$-糖苷键来降解肝素和硫 酸肝素(图 1). 乙酰肝素酶通过释放肝素寊糖参与多种 重要的生理过程, 如肿瘤细胞的浸润和转移 ${ }^{[6]}$ 、血管的 生成 ${ }^{[7]} 、$ 炎症 ${ }^{[8]}$ 、和平滑肌细胞的增生 ${ }^{[9]}$. 发展乙酰肝素 酶抑制剂用于抑制肿瘤细胞的增殖和转移、新血管的生 成和炎症的发生等, 是近来药物研究的一个热点课题.

2002 年, Okada 和 Yamada 等采用酸性条件裂解

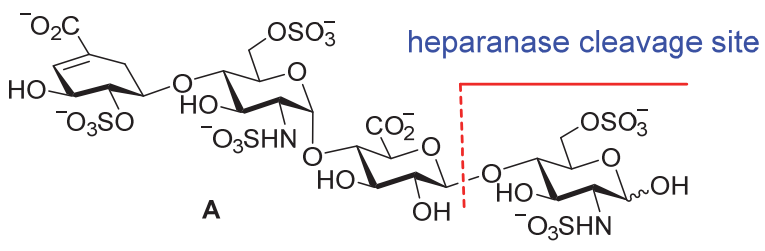

$\Delta \operatorname{HexA}(2 \mathrm{~S})-\mathrm{GIcN}(\mathrm{NS}, 6 \mathrm{~S})-\mathrm{GIcUA-GIcN}(\mathrm{NS}, 6 \mathrm{~S})$

图 1 肝素酶底物四糖 $\mathbf{A}$ 的结构

Figure 1 Structure of tetrasaccharide A relevant to the substrate of heparinase
肝素，分离得到一系列结构确定的肝素葟糖片段；随 后，测试肝素酶对这些肝素片段的水解活性，较系统、 明确地阐明了人源肝素酶所能识别的底物结构 ${ }^{[10]}$. 他 们发现四糖 $\mathbf{A}$ 和结构相关的六糖是肝素酶能较好识别 的底物. 四糖 $\mathbf{A}$ 结构为 $[\Delta \operatorname{HexUA}(2 \mathrm{~S})-\mathrm{GlcN}(\mathrm{NS}, 6 \mathrm{~S})-$ GlcUA-GlcN(NS,6S)](图 1), 由于其中只含一个酶切割 位点, 适合用作底物对肝素酶的水解活性进行定量分析. 他们的研究还发现, 肝素酶底物的结构中必须具有 -GlcN(NS,6S)-GlcUA-GlcN(NS,6S)-三糖片段. 其中葡萄 糖醛酸上的羧基、非还原端己胺糖 6 位羟基上的硫酸根 和还原端己胺糖氨基上的硫酸根至关重要. 非还原末端 己胺糖上的 $N$-硫酸根和还原端己胺糖 6 位上的 $O$-硫酸 根对于肝素酶的识别有促进作用, 而还原端己胺糖上 3 位羟基的硫酸根则对肝素酶的活性有抑制作用.

基于上述研究成果, 我们根据人源肝素酶所能较好 识别的肝素四糖片段 A 和最小的三糖片段(-GlcN(NS, 6S)-GlcUA-GlcN(NS,6S)-)的结构, 拟合成肝素臭糖 1 4(图 2), 以用于肝素酶底物选择性的进一步研究, 并发 展可用于肝素酶活测试的底物. 为了更好地模拟天然肝 素分子和方便地表征合成中间体和目标产物, 我们将四 糖 $\mathbf{A}$ 中非还原端的不饱和葡萄糖醛酸还原成天然的葡 萄糖醛酸, 并把还原端用 $\alpha$-甲苷锁定. 我们预期, 合成 寡糖 1 $\sim 4$ 与肝素酶作用后, 将被水解成二糖或三糖片 段和单糖 $\mathbf{B}$; 只要加入内标, 如氛代 $\alpha$-甲苷 $\mathbf{C}$, 即可通 过定量质谱测定 $\mathbf{B}$ 和 $\mathbf{C}$ 的比例来定量肝素酶的活性.

这里, 我们报道对肝素三糖和四糖 $1 \sim 4$ 的化学合 成.
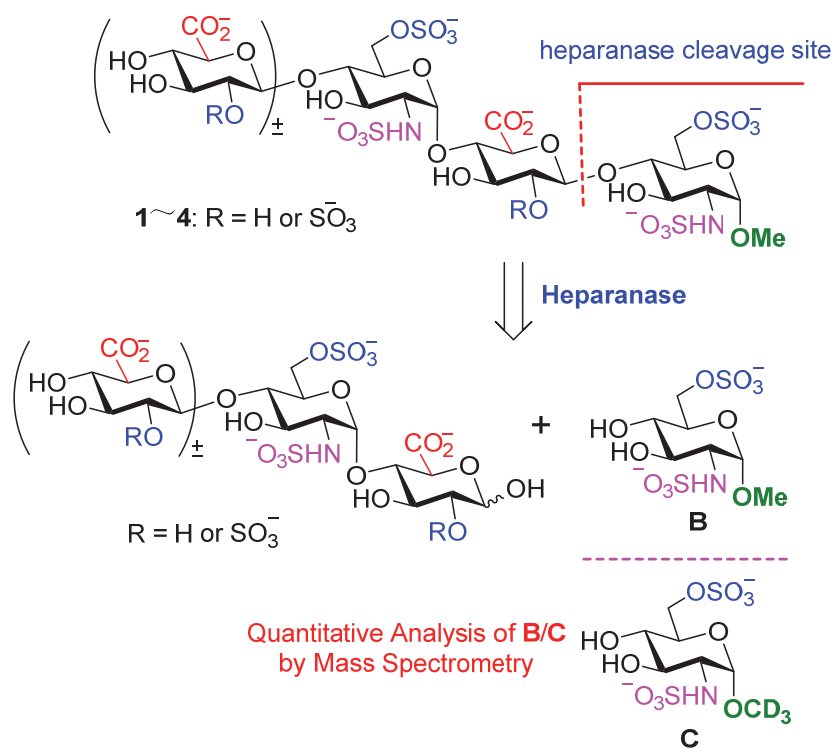

图 2 三糖和四糖 1 $\sim 4$ 的水解和肝素酶活性定量分析

Figure 2 Hydrolysis of tri- and tetrasaccharides $1 \sim \mathbf{4}$ and quantitative analysis of the activity of heparinase. 


\section{2 结果与讨论}

\section{1 逆合成分析}

如图 3 所示, 我们拟采取精简的保护基策略, 将需 要硫酸化的羟基用苯甲酰基(Bz)保护, 需要硫酸化的氨 基采用叠氮基 $\left(\mathrm{N}_{3}\right)$ 取代, 裸露的羟基和糖醛酸 6 位的羧 酸根采用苄基 $(\mathrm{Bn})$ 保护. 采用汇聚式的 $[1+2]$ 和 $[2+2]$ 糖苷化策略, 寡糖 $5 \sim 8$ 可以从单糖砌块 9 和二糖砌块 10 12 拼接制备; 我们拟采用直接脱水糖苷化方法来 立体选择性地构建其中关键的 $\alpha-(1 \rightarrow 4)$ 糖苷键 ${ }^{[11]}$.

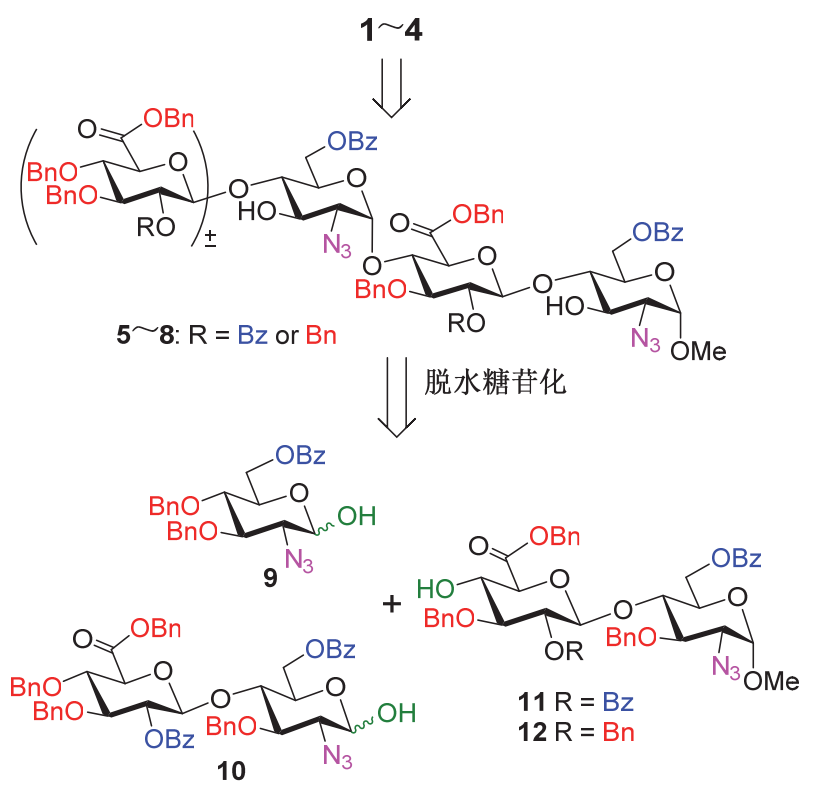

图 3 肝素酶底物三糖和四糖 $\mathbf{1} \sim \mathbf{4}$ 的逆合成分析

Figure 3 Retrosynthetic analysis of tri- and tetrasaccharides $1 \sim 4$

\section{2 二糖砌块的合成}

如图式 1 所示, 从已知二糖砌块 $10 \mathrm{~S}^{[12 b]}$ 出发, 采用 嗍烷四氢呋喃 $\left(\mathrm{BH}_{3} \cdot \mathrm{THF}\right) /$ 二丁基嗍三氟甲磺酸 $\left(\mathrm{Bu}_{2} \mathrm{~B} \cdot \mathrm{OTf}\right)$ 区域选择性的还原苄叉保护基, 裸露出 6 位 羟基 ${ }^{[13]}$. 对于 6 位羟基进行 2,2,6,6-四甲基哌啶-氮-氧化 物(TEMPO)/醋酸碘苯(BAIB)氧化 ${ }^{[14]}$, 随后, 苠酯化; 接 着, 采用四丁基氟化铵(TBAF)/醋酸(HOAc)条件, 脱除
异头位的叔丁基二甲基硅基(TBS)，以四步 77\%的收率， 制备得到二糖半缩醛 10 . 从已知二糖砌块 $11 S$ 和 $12 S^{[12 b]}$ 出发, 用醋酸水溶 $(\varphi$ 醀 $=80 \%$ ) 液脱除苠叉, 得到 4,6 -二羟基二糖化合物; 接着, 对 6 位伯羟基进行 TEMPO 氧 化和茮酯化, 顺利得到二糖受体 11 和 12. 在合成二糖 11 和 12 时, 裸露的 4 位羟基可以促进 TEMPO 氧化，该 反应在 $4.5 \mathrm{~h}$ 内完成. 而在合成化合物 $\mathbf{1 0}$ 时，底物中的 4 位羟基被茮基保护，相应的 TEMPO 氧化反应进行得非 常缓慢. 使用正常试剂量 $\left(n_{\mathrm{BAIB}} / n_{11 \mathrm{~S}}\right.$ or $12 \mathrm{~S}=2.5, n_{\mathrm{TEMPO}}$ $/ n_{11 \mathrm{~S}}$ or $\left.12 \mathrm{~s}=0.03\right)$, 过夜反应才能完全. 若增加醋酸碘苯 至 $n_{\mathrm{BAIB}} / n_{11 \mathrm{~S} \text { or } 12 \mathrm{~S}}=4$, 则 $3 \mathrm{~h}$ 即能反应完全.

\section{3 脱氧糖苷化方法构建 $\alpha-(1 \rightarrow 4)$ 糖苷键}

成功得到糖砌块之后, 我们准备尝试[2+2]糖苷化 反应，探索最佳反应条件来获得高产率和优秀的 $\alpha$ 选择 性. 糖苷化反应是聚糖合成中最重要的反应，糖化学家 已发展出多种高效的糖苷化方法，其中有很多糖苷化方 法被成功应用于肝素类寡糖的化学合成中 ${ }^{[15]}$. 常用的 糖苷化反应需要两步操作, 即先由糖基半缩醛进行 1 位 羟基衍生化得到糖基给体，再在促进剂活化下与亲核性 受体进行糖苷化反应. 与之相比, 异头位裸露的糖基半 缩醛直接进行脱水糖苷化反应，在形式上更加方便和高 效. 近年来, 多种脱水试剂组合的开发, 使得这种糖苷 化方法也得到了较大的发展 ${ }^{[11,16,17]} .2000$ 年, Gin 小组发 现在亚砜或者硫醚以及 $\mathrm{Tf}_{2} \mathrm{O}$ 的作用下可以顺利地完成 一步脱水糖苷化, 并且取得较好的 $\alpha$ 选择性 ${ }^{[17]}$. 随后, 荷兰莱顿大学的 van der Marel 等将这种脱水糖苷化方 法应用于肝素塞糖的合成 ${ }^{[18]}$, 采用“一锅法”合成策略, 以较好的 $\alpha$ 选择性得到了保护的肝素五糖. 几乎在相同 时间，我们也开展了类似的工作 ${ }^{[19]}$.

在这里，我们把该脱水糖苷化方法应用于目标寡糖 1 4 的合成. 首先将二糖半缩醛 10 溶于干燥的二氯甲 烷(DCM)中, 加入二苯亚砜 $\left(\mathrm{Ph}_{2} \mathrm{SO}\right) 、 2,4,6$-三叔丁基吡 啶(TTBP)和 $4 \AA$ 分子笁( $\mathrm{MS})$, 充分搅拌后, 冷却至 $-60{ }^{\circ} \mathrm{C}$, 慢慢滴加三氟甲磺酸酐 $\left(\mathrm{Tf}_{2} \mathrm{O}\right)$, 滴加完毕后升 至 $-40{ }^{\circ} \mathrm{C}$ 反应 $1 \mathrm{~h}$. 然后, 将受体 11 的二氯甲烷溶液滴
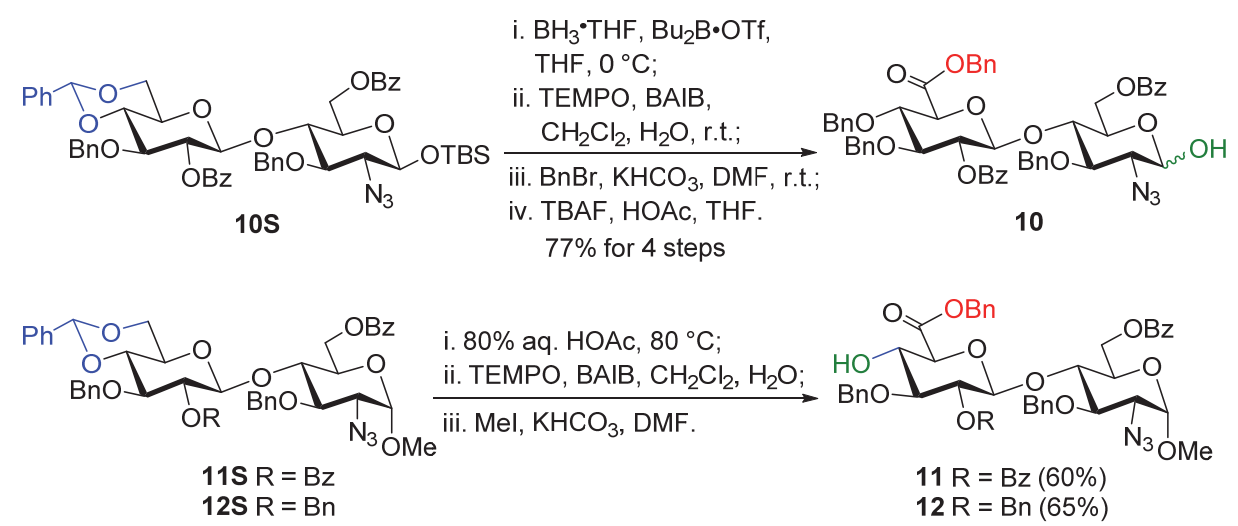

图式 1 二糖砌块 10 12 的合成

Scheme 1 Synthesis of disaccharides $\mathbf{1 0} \sim \mathbf{1 2}$ 
加进反应液, 最后自然升温至室温反应，薄层层析(TLC) 监测, 约 $3 \mathrm{~h}$ 后滴加三乙胺 $\left(\mathrm{NEt}_{3}\right)$ 淬灭反应. 该糖苷化反 应的选择性非常好, 只得到 $\alpha$ 产物, 但是, 产率只有 $32 \%$ (Table 1, entry 1). TCL 监测发现, 体系中出现一个 荧光很强、极性较小的副产物, 怀疑是给体 10 异头位发 生消除的糖烯产物. 但是, 该副产物不稳定, 柱层析分 离后即发生分解. 为了减少这一副产物的生成, 反应在 升温至 $-40{ }^{\circ} \mathrm{C}$ 后, 尝试只搅拌 $15 \mathrm{~min}$, 即加入受体 11 的二氯甲烷溶液. 结果糖苷化仍然只得到 $\alpha$ 产物, 但是, 产率反而下降至 22\%(Table 1, entry 2). 考虑到有时候用 甲苯做溶剂可以显著提高糖苷化反应的收率 ${ }^{[12 b]}$, 我们 更换溶剂为甲苯，确实，糖苷化产率提高到 $65 \%$, 而 $\alpha$ 选择性稍有下降 $(\alpha \beta=8.3 / 1.0)$ (Table 1, entry 3).

表 1 二糖 10 和 12 脱水糖苗化反应的条件优化

Table 1 Optimization of dehydrative glycosylation of disaccharides $\mathbf{1 0}$ and $\mathbf{1 2}$

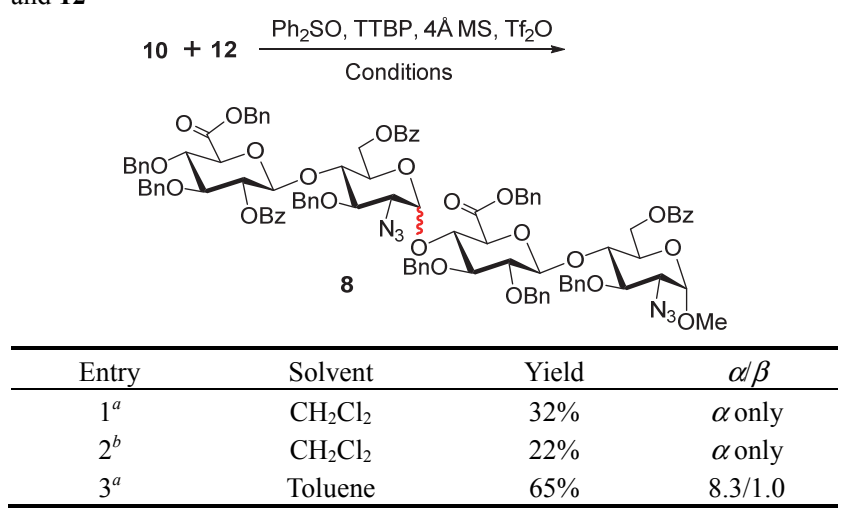

a 室温条件下, 将二糖 10, 二苯亚砜 $\left(\mathrm{Ph}_{2} \mathrm{SO}\right), 2,4,6$-三叔丁基嘧啶(TTBP)溶于溶剂中, 加入 $4 \AA$ 分子筛, 摚拌 $30 \mathrm{~min} ;-60{ }^{\circ} \mathrm{C}$ 下加入三氟甲磺酸䣶 $\left(\mathrm{Tf}_{2} \mathrm{O}\right)$, 升至 $-40{ }^{\circ} \mathrm{C}$ 反应 $1 \mathrm{~h}$; 加入二糖 12, 自然升至室温反应(约 $3 \mathrm{~h}$ ). ${ }^{b}$ 室温条件下, 将 $\mathbf{1 0}$, 二苯亚砜, 2,4,6-三 叔丁基嘧啶溶于溶剂中, 加入 $4 \AA$ 分子笁, 搅拌 $30 \mathrm{~min} ;-60{ }^{\circ} \mathrm{C}$ 条件下加入三氟甲磺 酸䣶, 升至 $-40{ }^{\circ} \mathrm{C}$ 反应 $15 \mathrm{~min}$; 加入 12 , 自然升至室温反应(约 $3 \mathrm{~h}$ )
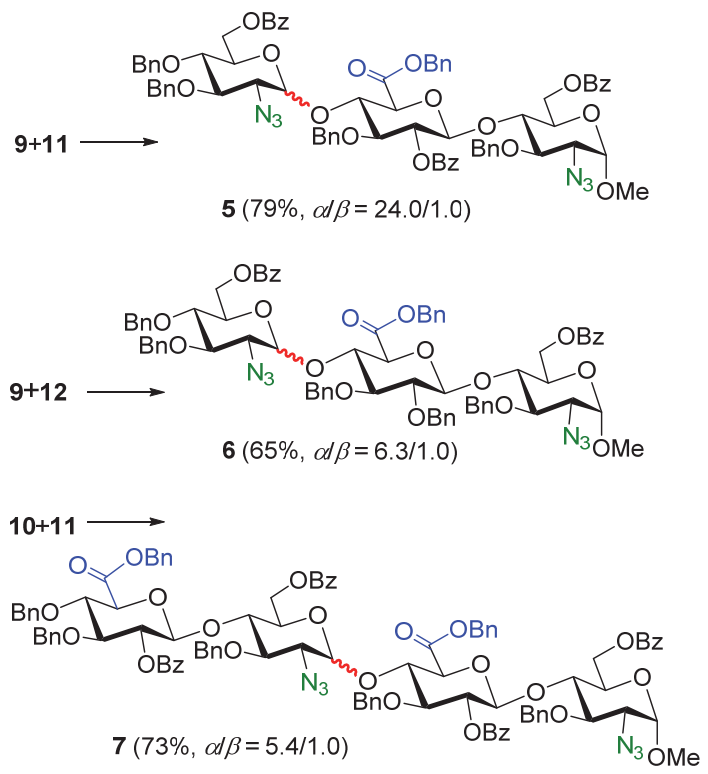

图式 2 脱水糖苷化合成三糖 5 和 $\mathbf{6}$ 和四糖 7

Scheme 2 Dehydrative glycosylation for the synthesis of tri- and tetrasaccharides $5 \sim 7$
随后，我们利用优化的脱水糖苷化条件 $\left(\mathrm{Ph}_{2} \mathrm{SO}\right.$, TTBP, $4 \AA \mathrm{MS}, \mathrm{Tf}_{2} \mathrm{O}$, toluene), 使用单糖给体 $\mathbf{9}^{[12 \mathrm{~b}]}$ 、二糖 给体 10 分别与二糖受体 11 和 12 进行糖苷化反应, 顺利 合成了三糖 5、6 和四糖 7(图式 2). 糖苷化反应的产率 都在 $65 \%$ 以上, $\alpha / \beta$ 选择性都高于 5.4/1. 值得一提的是, 该脱水糖苷化反应的 $\alpha$ 选择性优于采用 $N$-苯基三氟乙酰 亚胺酯给体对类似底物的糖苗化 ${ }^{[12]}$.

\section{4 保护基脱除和硫酸化方法的摸索}

肝素类寡糖的化学合成中的一大难点是需要对多 个羟基和氨基的完全硫酸化操作 ${ }^{[15]}$. 在肝素合成的文 献中, 脱保护和硫酸化的条件很多 ${ }^{[20]}$. 在成功构建了肝 素酶底物三糖和四糖骨架后, 我们尝试探索最优的保护 基脱除和硫酸化修饰的操作步骤.

水解脱除酯基保护基(Bn)和酰基保护基(Bz): 在文 献中 ${ }^{[15,20 a, 20 b, 21]}$, 一般采用甲酯保护糖醛酸的 6 位羧基, 为了防止碱性条件下脱除甲酯的同时发生糖醛酸的消 除反应，通常需要采用现制的氢过氧化锂 $(\mathrm{LiOOH})$ 将甲 酯和部分酯基脱除; 再加入氢氧化钾或者氢氧化钠水溶 液, 脱除剩余的酰基. 我们采用 $\mathrm{Bn}$ 作为糁基保护基, 避 免了上述繁琐操作. 直接采用 $3 \mathrm{~mol} / \mathrm{L}$ 氢氧化钾水溶液, 室温反应 $24 \mathrm{~h}$, 可以一步 $96 \%$ 的收率得到 $O$-硫酸化前体 13. 在这步反应中, 未检测到葡萄糖醛酸发生消除反应 的副产物.

$O$-硫酸化: $O$-硫酸化的一般条件有: $\mathrm{SO}_{3}$-pyridine, DMF, r.t., $>3 \mathrm{~h}^{[12 \mathrm{~b}, 20 \mathrm{a}, 21 \mathrm{a}, 21 \mathrm{~b}]} ; \mathrm{SO}_{3} \cdot \mathrm{Me}_{3} \mathrm{~N}$ 或者 $\mathrm{SO}_{3} \cdot \mathrm{Et}_{3} \mathrm{~N}$, DMF, $55{ }^{\circ} \mathrm{C}, 24 \mathrm{~h}^{[12 \mathrm{a}, 20 \mathrm{~b}, 20 \mathrm{~d}]}$; 氯磺酸, DMF, $8 \mathrm{~h}$; 也可以 采用吡啶作为溶剂. 氯磺酸较危险; 而 $\mathrm{SO}_{3} \cdot \mathrm{Me}_{3} \mathrm{~N}$ 或者 $\mathrm{SO}_{3} \cdot \mathrm{Et}_{3} \mathrm{~N}$ 作为硫酸化试剂的反应需要加热, 并且, 位阻 较大的糖醛酸 2 位羟基经常无法完全硫酸化. 经过摸索, 我们采用 $\mathrm{SO}_{3}$ •pyridine 作为硫酸化试剂, 无水 $\mathrm{DMF}$ 作为 溶剂, 反应 $5 \mathrm{~h}$, 便以定量的收率得到 $O$-硫酸化产物 14 . 该反应采用 TLC 监测(RP-18 反相板), 显示只有一个新 产物点生成. 后处理时, 先加入甲醇淬灭反应, 然后加 入三乙胺调节 $\mathrm{pH}$ 至碱性. 减压浓缩后, 经过 Sephadex LH-20 或者反相柱层析分离, 最后经 Dowex 50WX4 钠 离子柱交换, 便得到所需的 $O$-硫酸化产物.

叠氮还原：在文献中, 还原叠氮有很多方法: Staudinger 还原 ${ }^{[22]}$; 丙二硫醇还原 ${ }^{[23]}$; Lindlar 催化 ${ }^{[24]}$; Pd 催化 ${ }^{[25]} ; \mathrm{SmI}_{2}$ 还原 ${ }^{[26]}$ 等等. 我们经过多次尝试发现: 如 果采用 Lindlar 催化剂氢化, $101.3 \mathrm{kPa} \mathrm{H}_{2}$ 条件不足以将 寡糖 2 位叠氮基还原完全; 而用 $\mathrm{SmI}_{2}$ 还原叠氮的效果也 不理想; 丙二硫醇还原叠氮效果很好, 然而, 硫醇具有 恶臭气味, 后处理比较麻烦。综合考虑, 我们采用 Staudinger 还原叠氮, 具体操作是加入 $1 \mathrm{~mol} / \mathrm{L}$ 的三甲基 膦 $\left(\mathrm{PMe}_{3}\right)$ 的 THF 溶液和 $0.1 \mathrm{~mol} / \mathrm{L} \mathrm{NaOH}$ 水溶液, 反应过 夜. 反应结束后, 将体系旋干, 无需提纯, 可以直接进 行下一步氨基保护. 体系中加入碱的原因是 ${ }^{[27]}$ : 在 Staudinger 还原时, 由于兼酸根裸露, 会形成稳定的电 
荷分离中间体, 加入氢氧化钠溶液破坏这类中间体, 可 以顺利得到还原产物. 在这里选择三甲基膦作为还原试 剂, 是因为其沸点较低, 后处理简单, 直接旋干反应溶 剂，以便进行下一步 $N$-硫酸化.

$N$-硫酸化: 在肝素合成中, $N$-硫酸化的常用条件有: $\mathrm{SO}_{3} \bullet$ pyridine, $\mathrm{Et}_{3} \mathrm{~N}$, pyridine ${ }^{[21,28]}$ 和 $\mathrm{SO}_{3} \bullet$ pyridine, $\mathrm{Et}_{3} \mathrm{~N}$ 和 $0.1 \mathrm{~mol} / \mathrm{L} \mathrm{NaOH}, \mathrm{MeOH}^{[12 \mathrm{~b}, 20 \mathrm{a}]}$. 我们采用第二种条件, 这也是用于选择性 $N$-硫酸化的条件. 将上一步粗品溶 于甲醇中, 冰浴下分别加入三乙胺, $0.1 \mathrm{~mol} / \mathrm{L} \mathrm{NaOH}$, 将 体系调节到碱性, 每隔 $0.5 \mathrm{~h}$ 加入 $\mathrm{SO}_{3} \bullet$ pyridine(氨基摩尔 数的 5 倍), 分三批加入, 并且保持在 $0{ }^{\circ} \mathrm{C}$ 下反应约 $5 \mathrm{~h}$. 实验发现, 在这种方法操作下, $N$-硫酸化反应体系简单, TLC 监测原料点消失后, 旋干溶剂, 进行反相柱层析和 钠离子柱交换, 可以 $88 \%$ 的产率得到 $N$-硫酸化产物 $\mathbf{1 5}$.

还原氢化脱除苄基：还原氢化一般是肝素合成的 最后一步, 在几乎所有的文献中, 还原氢化脱除茮基这 一步都被忽视, 给出的实验操作非常简单, 例如 Seeberger 小组 $\left(\mathrm{Pd} / \mathrm{C}, 101.3 \mathrm{kPa} \mathrm{H}, \mathrm{MeOH} / \mathrm{H}_{2} \mathrm{O}\right)^{[21]}$; 黄雪飞小 组 $\left(\mathrm{Pd}(\mathrm{OH})_{2} / \mathrm{C} \text { or } \mathrm{Pd} / \mathrm{C}, 101.3 \mathrm{kPa} \mathrm{H}, \mathrm{MeOH} / \mathrm{H}_{2} \mathrm{O}\right)^{[20 \mathrm{~d}, 29]}$; Boons 小组两步策略 $\left(\mathrm{Pd} / \mathrm{C}, 101.3 \mathrm{kPa} \mathrm{H}, \mathrm{MeOH} / \mathrm{H}_{2} \mathrm{O}\right.$; $\left.\mathrm{Pd}(\mathrm{OH})_{2} / \mathrm{C}, 101.3 \mathrm{kPa} \mathrm{H}_{2}, \mathrm{H}_{2} \mathrm{O}\right)^{[20 \mathrm{a}]}$ 等等. 然而, 肝素鿒糖 中的氨基对于氢化反应有很大的影响, 采用上述常压氢 化条件, 甲醇和水作为溶剂, 反应从 $1 \mathrm{~d}$ 延长到 $3 \mathrm{~d}$, 苄 基仍然大量存在. 查阅台湾清华大学洪上程教授小组的 博士论文 ${ }^{[30]}$, 我们发现, 虽然发表文献中的氢化操作很 简单, 在实际操作中却都有类似的问题, 即无法将苄基 完全脱除. 洪上程小组的解决方法是：一、采取两次氢 化, 先加入质量为原料质量的两倍的 $\mathrm{Pd} / \mathrm{C}\left(w_{\mathrm{Pd}}=10 \%\right)$, 常压氢化 $1 \mathrm{~d}$, 之后过滤旋干, 再次投料反应 $1 \mathrm{~d}$, 可以 将苦基基本脱除; 二、尝试同时还原叠氮基团和茮基时, 他们注意到氨基的影响, 故添加了乙酸, 仍然效果不佳. 最后，通过篮选氢化条件发现，在 $344.7 \mathrm{kPa} \mathrm{H}$ 条件下， $\mathrm{Pd} / \mathrm{C}\left(w_{\mathrm{Pd}}=10 \%\right)$ 催化氢化可以将苄基和叠氮还原完全. 最近, 很多小组也开始尝试 $\mathrm{pH}=7$ 的缓冲水作为溶剂进 行还原氢化脱除茮基 ${ }^{[12 b, 20 b, 31]}$. 考虑到缓冲水作为溶剂, 会产生非常多的盐类物质，需要采用凝胶柱多次层析才 能去除, 增加了操作步骤. 我们最终决定采用高压氢化 条件 $\left(1013 \mathrm{kPa} \mathrm{H}, \mathrm{Pd}(\mathrm{OH})_{2} / \mathrm{C}\left(w_{\mathrm{Pd}}=10 \%\right), \mathrm{MeOH} / \mathrm{H}_{2} \mathrm{O}\right)$, 反应 $3 \mathrm{~d}$ 可以将茮基完全脱除, 以几乎定量的收率得到 最终的肝素四糖 3. 高压氢化这个方法的后处理简单, 只需要过滤除去钯催化剂, 即能快速得到最终的肝素宣 糖.

按照上述优化的保护基脱除和硫酸化的实验步骤, 我们顺利制备得到了另外三个目标寡糖 1, 2 和 4. 最后 五步的总收率分别为 54\%, 71\%和 52\%(图式 4). 肝素酶 底物三糖和四糖 1 4 的核磁和质谱数据与文献报道的 相符合 ${ }^{[12]}$.
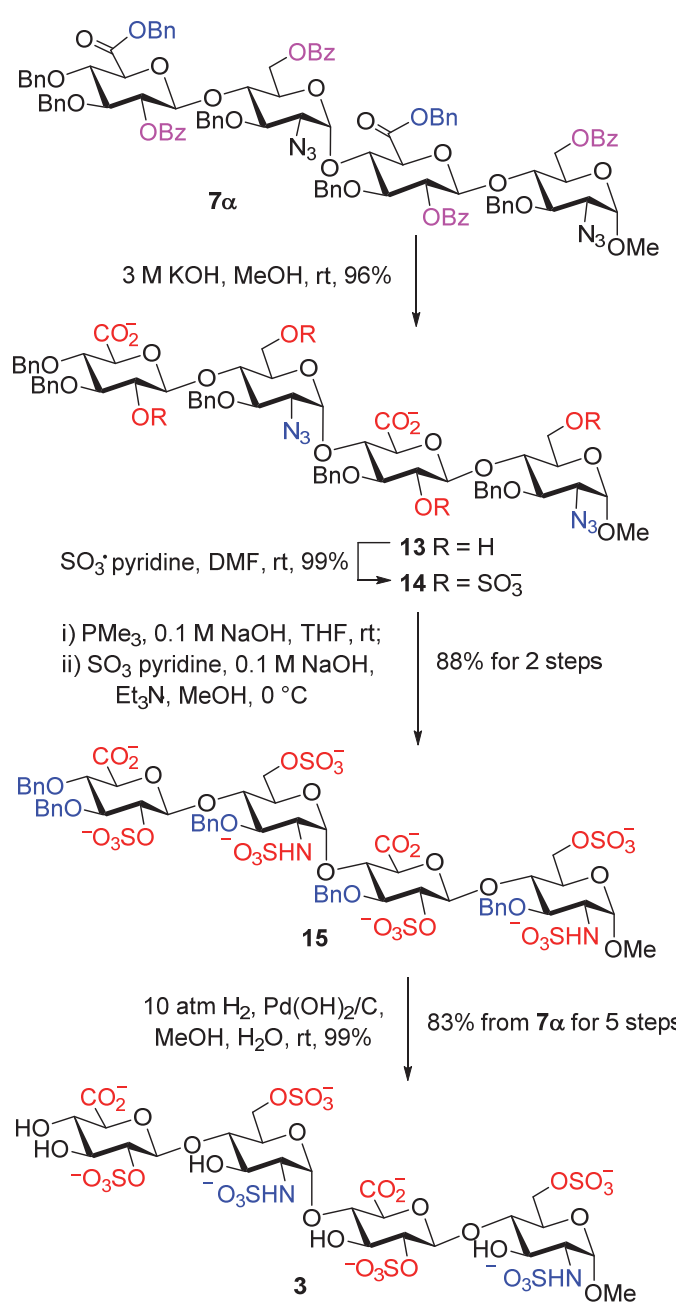

图式 3 四糖 3 的合成

Scheme 3 Synthesis of tetrasaccharide 3

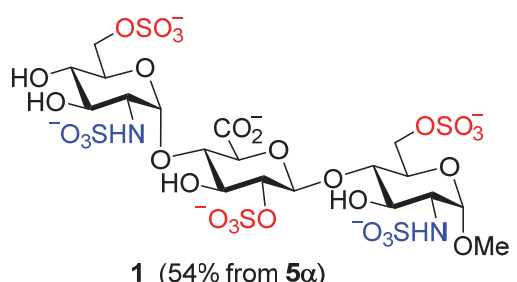

$1(54 \%$ from $5 \alpha)$

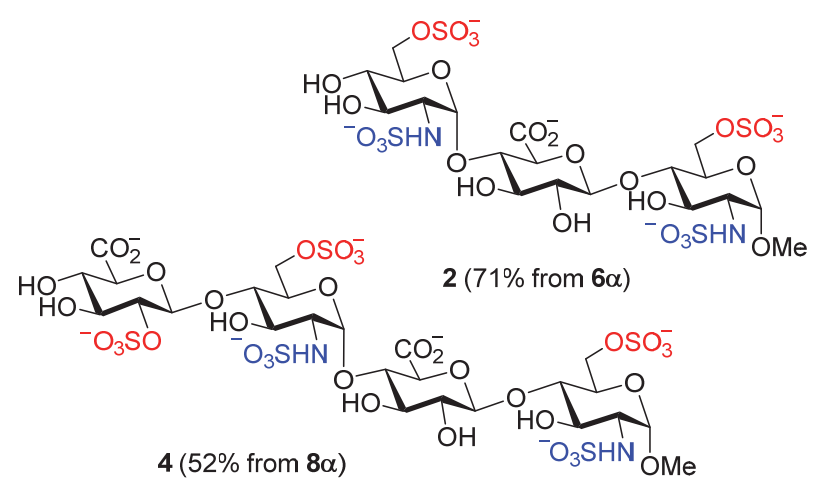

图式 4 三糖 1 和 2 和四糖 4 的合成

Scheme 4 Synthesis of trisaccharides 1 and $\mathbf{2}$ and tetrasaccharide 4 


\section{5 单糖底物的定量质谱分析方法的建立}

乙酰肝素酶是哺乳动物体内的内切葡萄糖醛酸水 解酶, 在肿瘤细胞的增殖与转移中起着非常重要的作用. 当人体中发生细胞的癌突, 在其癌变部位的乙酰肝素酶 的表达会发生异常. 定量质谱分析肝素酶水解能力的基 础是需要实现还原端单糖 $\mathbf{B}$ 与氞代标准物单糖 $\mathbf{C}^{[20 \mathrm{i}]}$ 的 定量质谱检测. 为此, 我们对于合成的单糖 $\mathbf{B}$ 和 $\mathbf{C}$ 进行 了不同比例下的定量质谱测试.

表 2 单糖 $\mathrm{B} / \mathrm{C}$ 的电喷雾质谱测试结果

Table 2 ESI Test for monosaccharide $\mathbf{B} / \mathbf{C}$

\begin{tabular}{|c|c|c|c|c|c|c|}
\hline $\begin{array}{c}\text { Sample } \\
\text { No. }\end{array}$ & $\begin{array}{c}\text { Molar } \\
\operatorname{ratio}(\mathbf{B} / \mathbf{C})\end{array}$ & $\begin{array}{c}\text { Observed } \\
m / z \text { for } \\
{[\mathrm{M}-}\end{array}$ & Intensity(B) & $\begin{array}{c}\text { Observed } \\
m / z \text { for } \\
{[\mathrm{M}-}\end{array}$ & Intensity $(\mathbf{C})$ & $\begin{array}{l}{ }_{b} \text { Intensity } \\
\operatorname{ratio}(\mathbf{B} / \mathbf{C})\end{array}$ \\
\hline & & $\mathrm{Na}]^{-}(\mathbf{B})^{a}$ & & $\mathrm{Na}]^{-}(\mathbf{C})$ & & \\
\hline 1 & $\mathrm{NA}^{c}$ & $\mathrm{NA}$ & NA & NA & NA & NA \\
\hline 2 & $9: 1$ & 374.13 & 49411 & 377.07 & 6486 & 7.6181 \\
\hline 3 & $8: 2$ & 374.27 & 77031 & 377.20 & 22613 & 3.4065 \\
\hline 4 & $7: 3$ & 374.27 & 65635 & 377.20 & 35878 & 1.8294 \\
\hline 5 & $6: 4$ & 374.27 & 49172 & 377.20 & 42720 & 1.1510 \\
\hline 6 & $5: 5$ & 374.20 & 31805 & 377.13 & 42525 & 0.7479 \\
\hline 7 & $4: 6$ & 374.27 & 27158 & 377.13 & 54446 & 0.4988 \\
\hline 8 & $3: 7$ & 374.33 & 22614 & 377.20 & 84840 & 0.2665 \\
\hline
\end{tabular}
$[\mathrm{M}-\mathrm{Na}]^{-}$is $377.32 ;{ }^{c}$ Not available.

根据不同比例单糖的定量质谱丰度比做出量化曲 线图(图 4), 建立了最佳的回归曲线和回归方程 $y=$ $0.8600 x-0.1085$, 相关性系数 $R^{2}=0.9996$. 这一结果为 定量测试肝素酶水解能力打下坚实的基础.



图 4 单糖 $\mathrm{B} / \mathrm{C}$ 的量化曲线

Figure 4 The quantification curve of monosaccharide $\mathbf{B} / \mathbf{C}$

\section{3 结论}

我们报道了一条高效的合成肝素寡糖的路线: 采用 苯甲酰基保护待硫酸化的羟基、苄基保护羧基和裸露的 羟基、叠氮基保护氨基; 应用脱水糖苷化方法高效构建 关键的 GlcN-(1 $\alpha \rightarrow 4)-G l c A$ 糖苷键; 采用标准的保护基 脱除和硫酸化操作, 高效、便捷地获得肝素酶底物三糖 和四糖 1 4. 最后五步操作总收率超过 $52 \%$. 这些寡糖 的合成为我们进一步研究肝素酶的底物选择性, 并建立
一套肝素酶活性定量测试方法打下了基础. 而高效灵敏 的测活方法可用于肝素酶抑制剂的篮选和肿瘤等相关 疾病的诊断.

\section{4 实验部分}

未特别注明的商业化试剂并未进行进一步纯化. 常 用的溶剂使用微波炉活化的 $4 \AA$ 分子篮进行干燥. 核磁 共振数据由 Bruker AMX-300 和 BrukerAV-400 型核磁共 振仪测定(以 TMS 作为内标); 高分辨质谱由 HP5989A 和 VG Quattro $\mathrm{MS} / \mathrm{MS}$ 质谱仪测定; 比旋光度由 Perkin-Elmer $241 \mathrm{MC}$ 型自动比旋光仪测定, 光源为钠 源, $589 \mathrm{~nm}$; 板层析监测采用 $10 \%$ 浓硫酸乙醇加热显色 或是 UV254 $\mathrm{nm}$ 显色.

(Benzyl 2-O-benzoyl-3,4-di- $O$-benzyl- $\beta$ - $D$-glucopyranosyluronate)-(1 $\rightarrow 4)$-2-azido-6- $O$-benzoyl-3- $O$ benzyl-2-deoxy- $\alpha / \beta$ - $D$-glucopyranose(10):

二糖化合物 $13^{[12 \mathrm{~b}]}(2.6 \mathrm{~g}, 2.7 \mathrm{mmol})$ 溶于干燥的四氢 呋喃 $(3 \mathrm{~mL})$, 冰浴冷却, 加入 $\mathrm{BH}_{3} \cdot \mathrm{THF}(1 \mathrm{~mol} / \mathrm{L}, 27 \mathrm{~mL})$, 搅拌 $5 \mathrm{~min}$, 加入 $\mathrm{Bu}_{2} \mathrm{~B} \cdot \mathrm{OTf}$ 的二氯甲烷溶液 $(1 \mathrm{~mol} / \mathrm{L}$, $5.4 \mathrm{~mL}$ ). 冰浴下反应 $3 \mathrm{~h}, \mathrm{TLC}$ 显示反应完全. 加入三乙 胺(1.5 mL)后, 缓慢滴加甲醇淬灭反应, 减压浓缩, 再分 别加入甲醇稀释, 浓缩三次. 柱层析 $\left(V_{\text {石油醚 }}: V_{\text {乙酸乙酯 }}=6\right.$ : $1)$ 得 到 tert-Butyldimethylsilyl(2-O-benzoyl-3,4-Obenzyl- $\beta$-D-glucopyranosyl)-( $1 \rightarrow 4)$-2-azido-6-O-benzoyl3-O-benzyl-2-deoxy- $\beta$-D-glucopyranoside 无色糖浆( $2.4 \mathrm{~g}$, $2.5 \mathrm{mmol})$, 产率 $93 \% . R_{f}=0.45\left(V_{\text {石油梄 }}: V_{\text {乙酸乙酯 }}=4: 1\right)$; ${ }^{1} \mathrm{H} \operatorname{NMR}\left(400 \mathrm{MHz}, \mathrm{CDCl}_{3}\right) \delta: 8.01(\mathrm{~d}, 2 \mathrm{H}, J=6.8 \mathrm{~Hz})$, $7.91(\mathrm{~d}, 2 \mathrm{H}, J=7.2 \mathrm{~Hz}), 7.57(\mathrm{t}, 1 \mathrm{H}, J=7.2 \mathrm{~Hz}), 7.44 \sim$ $7.24(\mathrm{~m}, 15 \mathrm{H}), 7.12 \sim 7.08(\mathrm{~m}, 5 \mathrm{H}), 5.29(\mathrm{t}, 1 \mathrm{H}, J=9.2$ $\mathrm{Hz}), 4.95(\mathrm{~d}, 1 \mathrm{H}, J=11.2 \mathrm{~Hz}), 4.83 \sim 4.70(\mathrm{~m}, 3 \mathrm{H}), 4.62 \sim$ $4.58(\mathrm{~m}, 3 \mathrm{H}), 4.47 \sim 4.42(\mathrm{~m}, 2 \mathrm{H}), 4.35(\mathrm{dd}, 1 \mathrm{H}, J=10.8$ $\mathrm{Hz}, 6.0 \mathrm{~Hz}), 3.80 \sim 3.74(\mathrm{~m}, 2 \mathrm{H}), 3.63 \sim 3.58(\mathrm{~m}, 2 \mathrm{H})$, $3.47 \sim 3.43(\mathrm{~m}, 1 \mathrm{H}), 3.39 \sim 3.30(\mathrm{~m}, 3 \mathrm{H}), 3.26 \sim 3.22(\mathrm{~m}$, $1 \mathrm{H}), 0.83(\mathrm{~s}, 9 \mathrm{H}), 0.057$ (s, 3H), 0.034 (s, 3H); HRMS (ESI) calcd for $\mathrm{C}_{53} \mathrm{H}_{61} \mathrm{~N}_{3} \mathrm{O}_{12} \mathrm{SiNa}$ : 982.3917; Found: 982.3942 .

将上一步得到的无色糖浆(1.53 g, $1.59 \mathrm{mmol})$ 溶于 二氯甲烷和水的混合溶液 $(15 \mathrm{~mL}, V: V=2: 1)$, 加入 TEMPO (5 mg, $0.03 \mathrm{mmol}$ )和BAIB (1.3 g, $4.0 \mathrm{mmol})$. 室 温下剧烈搅拌过夜(约 $12 \mathrm{~h}$ ), TLC显示反应完全. 饱和 $\mathrm{NaHSO}_{3}$ 水溶液淬灭反应，二氯甲烷稀释，分层，收集有 机相. 加入 $5 \%$ 的稀盐酸酸化水层, 二氯甲烷萃取水相 三次, 合并有机相, 无水硫酸钠干燥, 过滤, 用旋转蒸 发仪旋干溶剂, 得到粗产品tert-Butyldimethylsilyl (2-O-benzoyl-3,4-O-benzyl- $\beta$ - $D$-glucopyranosyluronate)$(1 \rightarrow 4)$-2-azido-6-O-benzoyl-3-O-benzyl-2-deoxy- $\beta$ - $D$ glucopyranoside为浅黄色糖浆.

将上一步的浅黄色糖浆溶解于无水 DMF (15 mL) 
中, 加入 $\mathrm{KHCO}_{3}(640 \mathrm{mg}, 6.4 \mathrm{mmol})$, 冰浴冷却, 加入 苠溴 $(0.3 \mathrm{~mL}, 2.4 \mathrm{mmol})$. 恢复室温, 反应过夜, TLC 显 示反应完成. 加入水和乙酸乙酯稀释, 分出有机相, 水 相用乙酸乙酯萃取三次, 合并有机相, 饱和食盐水溶液 洗一次, 无水硫酸钠干燥. 柱层析纯化 $\left(V_{\text {石油梄 }}: V_{\text {乙酸乙酯 }}=\right.$ $5: 1$ )得到 tert-Butyldimethylsilyl(benzyl 2-O-benzoyl3,4-O-benzyl- $\beta$ - $D$-glucopyranosyluronate)-( $1 \rightarrow 4)$-2-azido6- $O$-benzoyl-3- $O$-benzyl-2-deoxy- $\beta$ - $D$-glucopyranoside 白 色固体 $(1.5 \mathrm{~g}, 1.4 \mathrm{mmol})$, 两步产率 $88 \%$.

称取上一步白色固体 $(410 \mathrm{mg}, 0.39 \mathrm{mmol})$ 溶于四氢 呋喃(12 mL)和醋酸 $(0.07 \mathrm{~mL}, 1.2 \mathrm{mmol})$, 室温磁力搅拌 下，加入 TBAF的四氢呋喃溶液 $(1 \mathrm{~mol} / \mathrm{L}, 0.8 \mathrm{~mL}, 0.8$ $\mathrm{mmol})$. 自然升至室温反应 $1.5 \mathrm{~h}, \mathrm{TLC}$ 跟踪监测反应完全 后, 加入二氯甲烷稀释, 水洗, 饱和碳酸氢钠溶液洗, 食盐水洗, 无水硫酸钠干燥. 过滤, 浓缩, 柱层析 ( $V_{\text {石油瞵 }}$ : $\left.V_{\text {乙酸乙酯 }}=2: 1\right)$, 得无色糖浆 $\mathbf{1 0}$ (344 mg, $\left.0.4 \mathrm{mmol}\right)$, 收率 94\%. ESI-MS $(\mathrm{m} / \mathrm{z}): 891.8\left[\mathrm{M}+\mathrm{NH}_{4}\right]^{+}, 896.7[\mathrm{M}+\mathrm{Na}]^{+}$.

Methyl(benzyl 2-O-benzoyl-3-O-benzyl- $\beta$ - $D$-glucopyranosyluronate)-( $\rightarrow 4)$-2-azido-6- $O$-benzoyl-3- $O$-ben zyl-2-deoxy- $\alpha$ - $D$-glucopyranoside(11):

二糖化合物 $14^{[12 \mathrm{~b}]}(1.2 \mathrm{~g}, 1.6 \mathrm{mmol})$ 溶于冰乙酸 $(20$ $\mathrm{mL}$ )中, 加热至 $80{ }^{\circ} \mathrm{C}$, 慢慢滴加入 $5 \mathrm{~mL} \mathrm{H}_{2} \mathrm{O}$, 继续回 流反应 $2 \mathrm{~h}$, TLC 检测反应完全, 冷至室温, 旋干, 柱层 析 $\left(V\right.$ 石油瞵 $\left.: V_{\text {乙酸乙酯 }}=1: 1\right)$ 得到脱除苠叉的无色糖浆 $(1.0$ g, $1.4 \mathrm{mmol})$, 产率 96\%. ${ }^{1} \mathrm{H} \mathrm{NMR}\left(400 \mathrm{MHz}, \mathrm{CDCl}_{3}\right) \delta$ : $8.10(\mathrm{~d}, 2 \mathrm{H}, J=6.8 \mathrm{~Hz}), 7.95$ (d, 2H, $J=6.8 \mathrm{~Hz}), 7.59$ (t, $1 \mathrm{H}, J=7.2 \mathrm{~Hz}), 7.49 \sim 7.15(\mathrm{~m}, 15 \mathrm{H}), 5.31(\mathrm{t}, 1 \mathrm{H}, J=8.8$ $\mathrm{Hz}), 5.04$ (d, 1H, $J=10.8 \mathrm{~Hz}), 4.82(\mathrm{~d}, 1 \mathrm{H}, J=10.8 \mathrm{~Hz})$, $4.71 \sim 4.38(\mathrm{~m}, 6 \mathrm{H}), 3.94 \sim 3.61(\mathrm{~m}, 6 \mathrm{H}), 3.44 \sim 3.41(\mathrm{dd}$, $1 \mathrm{H}, J=3.6,9.6 \mathrm{~Hz}), 3.34(\mathrm{~s}, 3 \mathrm{H}), 3.34 \sim 3.16(\mathrm{~m}, 2 \mathrm{H})$, $2.54 \sim 2.52(\mathrm{~m}, 1 \mathrm{H}), 1.87 \sim 1.83(\mathrm{~m}, 2 \mathrm{H}) ;{ }^{13} \mathrm{C}$ NMR $(100$ $\left.\mathrm{MHz}, \mathrm{CDCl}_{3}\right) \delta: 166.1,165.0,138.2,137.7,133.4,129.8$, $129.5,129.2,128.7,128.6,128.5,128.0,127.93,127.90$, 127.4, 101.0, 98.4, 82.7, 78.1, 77.7, 75.7, 75.3, 74.8, 73.9, 70.6, 68.9, 63.2, 62.6, 62.0, 55.4; HRMS (MALDI/DHB) calcd for $\mathrm{C}_{41} \mathrm{H}_{43} \mathrm{~N}_{3} \mathrm{O}_{12} \mathrm{Na}$ : 792.2739; Found: 792.2735.

将上一步无色糖浆 $(1.0 \mathrm{~g}, 1.4 \mathrm{mmol})$ 溶于二氯甲烷 和水的混合溶液 $(15 \mathrm{~mL}, V: V=2: 1)$, 加入 $\mathrm{TEMPO}(4$ $\mathrm{mg}, 0.03 \mathrm{mmol}), \mathrm{BAIB}(1.0 \mathrm{~g}, 3.2 \mathrm{mmol})$, 室温下剧烈摚 拌 $4.5 \mathrm{~h}, \mathrm{TLC}$ 显示反应完全. 饱和 $\mathrm{NaHSO}_{3}$ 水溶液淬灭 反应, 二氯甲烷稀释, 分层, 收集有机相. 加入质量分 数 $5 \%$ 的稀盐酸酸化水层, 二氯甲烷萃取水相三次, 合 并有机相, 无水硫酸钠干燥, 过滤, 用旋转蒸发仪旋干 溶 剂, 得 到 Methyl(2-O-benzoyl-3-O-benzyl- $\beta$ - $D$ glucopyranosyluronate)-( $1 \rightarrow 4)-2$-azido-6- $O$-benzoyl-3- $O$ benzyl-2-deoxy- $\alpha$ - $D$-glucopyranoside 粗产品为浅黄色糖 浆.

上一步得到的糖浆溶解于 $\operatorname{DMF}(12 \mathrm{~mL})$ 中, 加入 $\mathrm{KHCO}_{3}(0.5 \mathrm{~g}, 5.1 \mathrm{mmol})$, 冰浴冷却, 加入苄溴 $(0.23 \mathrm{~mL}$,
$1.9 \mathrm{mmol})$, 恢复室温反应过夜. TLC显示反应完成. 加 入水和乙酸乙酯稀释, 分出有机相, 水相用乙酸乙酯萃 取三次, 合并有机相, 饱和食盐水溶液洗一次, 无水硫 酸钠干燥. 柱层析纯化 ( $V_{\text {石油瞵 }}: V_{\text {乙酸乙醌 }}=4: 1$ ) 得到化合 物 11 (0.7 g, $0.8 \mathrm{mmol})$, 为无色糖浆, 两步产率 $62 \%$. $[\alpha]_{\mathrm{D}}^{25}=80.1\left(c\right.$ 1.0, $\left.\mathrm{CHCl}_{3}\right) ;{ }^{1} \mathrm{H} \mathrm{NMR}\left(400 \mathrm{MHz}, \mathrm{CDCl}_{3}\right) \delta$ : $8.02(\mathrm{~d}, 2 \mathrm{H}, J=7.6 \mathrm{~Hz}), 7.88(\mathrm{~d}, 2 \mathrm{H}, J=7.2 \mathrm{~Hz}), 7.52$ (t, $1 \mathrm{H}, J=7.6 \mathrm{~Hz}), 7.45(\mathrm{t}, 1 \mathrm{H}, J=7.2 \mathrm{~Hz}), 7.38 \sim 7.26(\mathrm{~m}$, $14 \mathrm{H}), 7.12(\mathrm{~s}, 5 \mathrm{H}), 5.33(\mathrm{t}, 1 \mathrm{H}, J=8.8 \mathrm{~Hz}), 5.09 \sim 4.95(\mathrm{~m}$, $3 \mathrm{H}), 4.78 \sim 4.65(\mathrm{~m}, 5 \mathrm{H}), 4.49 \sim 4.45(\mathrm{~m}, 1 \mathrm{H}), 4.35(\mathrm{dd}$, $1 \mathrm{H}, J=12.0 \mathrm{~Hz}, 4.0 \mathrm{~Hz}), 4.06(\mathrm{t}, 1 \mathrm{H}, J=9.6 \mathrm{~Hz}), 3.97 \sim$ $3.90(\mathrm{~m}, 2 \mathrm{H}), 3.78 \sim 3.76(\mathrm{~m}, 1 \mathrm{H}), 3.71(\mathrm{~d}, 1 \mathrm{H}, J=10.0$ $\mathrm{Hz}), 3.64(\mathrm{t}, 1 \mathrm{H}, J=9.2 \mathrm{~Hz}), 3.33 \sim 3.30(\mathrm{~m}, 4 \mathrm{H}), 2.87(\mathrm{bs}$, $1 \mathrm{H}) ;{ }^{13} \mathrm{C} \mathrm{NMR}\left(100 \mathrm{MHz}, \mathrm{CDCl}_{3}\right) \delta: 168.39,165.97$, $164.96,138.25,137.68,134.86,133.39,133.31,129.85$, $129.56,129.53,129.18,128.64,128.59,128.51,128.34$, $128.29,127.99,127.60,101.14,98.51,80.97,77.96,77.79$, $75.33,74.75,74.44,73.24,72.14,68.69,67.51,63.25$, 62.55, 55.41; HRMS (ESI) calcd for $\mathrm{C}_{48} \mathrm{H}_{47} \mathrm{~N}_{3} \mathrm{O}_{13} \mathrm{Na}$ : 896.3001; Found: 896.3040.

Methyl(benzyl 2,3- $O$-benzyl- $\beta$ - $D$-glucopyranosyluronate)-( $\rightarrow 4)$-2-azido-3- $O$-benzyl-2-deoxy-6-O-benzoyl - $\alpha$ - $D$-glucopyranoside(12):

二糖化合物 $\mathbf{1 5}^{[12 \mathrm{~b}]}(0.9 \mathrm{~g}, 1.0 \mathrm{mmol})$ 溶于冰乙酸 $(10$ $\mathrm{mL}$ )中, 加热至 $80{ }^{\circ} \mathrm{C}$, 慢慢滴加入 $6 \mathrm{~mL} \mathrm{H} \mathrm{H}_{2} \mathrm{O}$, 继续回 流反应 $2 \mathrm{~h}$, TLC 检测反应完全, 冷至室温, 旋干, 柱层 析 $\left(V_{\mathrm{PE}}: V_{\mathrm{EA}}=1: 1\right)$ 得到脱除苄叉的无色糖浆(0.65 g, $0.86 \mathrm{mmol})$, 产率 $82 \% . \mathrm{ESI}-\mathrm{MS}(\mathrm{m} / \mathrm{z}): 773.5\left[\mathrm{M}+\mathrm{NH}_{4}\right]^{+}$, $778.3[\mathrm{M}+\mathrm{Na}]^{+}$.

将上一步无色糖浆(0.62 $\mathrm{g}, 0.82 \mathrm{mmol})$ 溶于二氯甲 烷和水的混合溶液 (15 mL, $V: V=2: 1)$, 加入 TEMPO(3 mg, $0.02 \mathrm{mmol}), \mathrm{BAIB}(0.66 \mathrm{~g}, 2.05 \mathrm{mmol})$, 室 温下剧烈摚拌 $4.5 \mathrm{~h}$, TLC 显示反应完全. 饱和 $\mathrm{NaHSO}_{3}$ 水溶液淬灭反应，二氯甲烷稀释，分层，收集有机相. 加入质量分数 $5 \%$ 的稀盐酸酸化水层，二氯甲烷萃取水 相三次, 合并有机相, 无水硫酸钠干燥, 过滤, 用旋转 蒸发仪旋干溶剂, 得到粗产品 $\operatorname{Methyl}(2,3-O$-benzyl- $\beta-D$ glucopyranosyluronate)-( $1 \rightarrow 4)$-2-azido-3-O-benzyl-2deoxy-6- $O$-benzoyl- $\alpha-D$-glucopyranoside 为浅黄色糖浆.

上一步得到的糖浆溶解于 $\operatorname{DMF}(10 \mathrm{~mL})$ 中, 加入 $\mathrm{KHCO}_{3}(0.33 \mathrm{~g}, 3.3 \mathrm{mmol})$, 冰浴冷却, 加入苠溴 $(0.15$ $\mathrm{mL}, 1.23 \mathrm{mmol})$, 恢复室温反应过夜. TLC显示反应完成. 加入水和乙酸乙酯稀释, 分出有机相, 水相用乙酸乙酯 萃取三次，合并有机相，饱和食盐水溶液洗一次，无水 硫酸钠干燥. 柱层析纯化 $\left(V_{\mathrm{PE}}: V_{\mathrm{EA}}=4: 1\right)$ 得到化合物 12(0.6 g, $0.65 \mathrm{mmol})$, 为无色糖浆, 两步产率 79\%. ${ }^{1} \mathrm{H}$ NMR (400 MHz, $\left.\mathrm{CDCl}_{3}\right) \delta: 7.95(\mathrm{~d}, 2 \mathrm{H}, J=7.6 \mathrm{~Hz}), 7.49$ $(\mathrm{t}, 1 \mathrm{H}, J=7.2 \mathrm{~Hz}), 7.41 \sim 7.25(\mathrm{~m}, 22 \mathrm{H}), 5.09 \sim 4.98(\mathrm{~m}$, $3 \mathrm{H}), 4.87 \sim 4.79(\mathrm{~m}, 4 \mathrm{H}), 4.73 \sim 4.66(\mathrm{~m}, 3 \mathrm{H}), 4.57(\mathrm{~d}, 1 \mathrm{H}$, 
$J=6.8 \mathrm{~Hz}), 4.44 \sim 4.39(\mathrm{dd}, 1 \mathrm{H}, J=12.4 \mathrm{~Hz}, 4.8 \mathrm{~Hz}), 4.02$ $(\mathrm{t}, 1 \mathrm{H}, J=9.2 \mathrm{~Hz}), 3.94 \sim 3.85(\mathrm{~m}, 2 \mathrm{H}), 3.78(\mathrm{dd}, 1 \mathrm{H}, J=$ $10.0 \mathrm{~Hz}, 3.2 \mathrm{~Hz}), 3.69$ (d, $1 \mathrm{H}, J=9.6 \mathrm{~Hz}), 3.45 \sim 3.42(\mathrm{~m}$, $5 \mathrm{H}), 3.36$ (dd, 1H, $J=10.4 \mathrm{~Hz}, 3.6 \mathrm{~Hz}), 2.67$ (d, 1H, $J=$ $2.4 \mathrm{~Hz}$ ); HRMS (ESI) calcd for $\mathrm{C}_{48} \mathrm{H}_{49} \mathrm{~N}_{3} \mathrm{O}_{12} \mathrm{Na}$ : 882.3209; Found: 882.3228 .

General Procedure A：脱水糖苷化方法具体步骤：

给体(1 equiv., $0.02 \mathrm{~mol} / \mathrm{L}$ in Tol)溶于适量干燥甲苯 中, 加入 $\mathrm{Ph}_{2} \mathrm{SO}$ (2.8 equiv.), TTBP (3.0 equiv.), $4 \AA \mathrm{MS}$, 室温下摚拌 0.5 h. 降温至 $-60{ }^{\circ} \mathrm{C}$, 滴加入 $\mathrm{Tf}_{2} \mathrm{O}(1.4$ equiv.), 升温至 $-40{ }^{\circ} \mathrm{C}$ 反应 $1 \mathrm{~h}$ 后, 滴加入受体(1.5 2.0 equiv. $)$ 的甲苯溶液 $(0.1 \mathrm{~mol} / \mathrm{L})$, 然后自然升温到室温 反应. 大约 1 到 $3 \mathrm{~h}$ 后, $\operatorname{TLC}\left(V_{\mathrm{Tol}}: V_{\mathrm{EA}}=20: 1\right)$ 检测反应 完全. 加入三乙胺淬灭反应. 硅藻土过滤. 滤液先后用 饱和 $\mathrm{NaHCO}_{3}$ 溶液, $\mathrm{NaCl}$ 溶液洗涤, 无水 $\mathrm{Na}_{2} \mathrm{SO}_{4}$ 干燥. 浓缩后通过柱层析(甲苯/乙酸乙酯)提纯可以分离出 $\alpha / \beta$ 异构体。

Methyl( 2-azido-3,4-O-benzyl-2-deoxy-6-O-benzoyl - $\alpha$-D-glucopyranosyl)-(1 $\rightarrow 4)$-(benzyl 2 - $O$-benzoyl-3- $O$ benzyl- $\beta$ - $D$-glucopyranosyluronate)-(1 $\rightarrow 4)$-2-azido-3$O$-benzyl-2-deoxy-6- $O$-benzoyl- $\alpha$ - $D$-glucopyranoside(5):

Procedure A: 给体 $9(81 \mathrm{mg}, 0.165 \mathrm{mmol})$, 受体 $11(290 \mathrm{mg}, 0.330 \mathrm{mmol})$; 柱层析 $\left(V_{\mathrm{Tol}}: V_{\mathrm{EA}}=30: 1\right)$, 三 糖产物 5: $175 \mathrm{mg}, 79 \%, \alpha: \beta=24.0: 1.0$.

$[\alpha]_{\mathrm{D}}^{23}=64.5\left(c 1.0, \mathrm{CHCl}_{3}\right) ;{ }^{1} \mathrm{H} \mathrm{NMR}(400 \mathrm{MHz}$, $\left.\mathrm{CDCl}_{3}\right) \delta: 8.08(\mathrm{~d}, 2 \mathrm{H}, J=7.2 \mathrm{~Hz}), 8.04(\mathrm{~d}, 2 \mathrm{H}, J=7.2 \mathrm{~Hz})$, $7.94(\mathrm{~d}, 2 \mathrm{H}, J=7.2 \mathrm{~Hz}), 7.52 \sim 7.14(\mathrm{~m}, 34 \mathrm{H}), 5.49 \sim 5.45$ $(\mathrm{m}, 2 \mathrm{H}), 5.11 \sim 5.00(\mathrm{~m}, 3 \mathrm{H}), 4.89 \sim 4.39(\mathrm{~m}, 14 \mathrm{H}), 3.99 \sim$ $3.91(\mathrm{~m}, 4 \mathrm{H}), 3.84(\mathrm{t}, 1 \mathrm{H}, J=9.2 \mathrm{~Hz}), 3.77 \sim 3.75(\mathrm{~m}, 2 \mathrm{H})$, $3.66(\mathrm{t}, 1 \mathrm{H}, J=9.6 \mathrm{~Hz}), 3.37 \sim 3.30(\mathrm{~m}, 5 \mathrm{H}) ;{ }^{13} \mathrm{C} \mathrm{NMR}$ $\left(100 \mathrm{MHz}, \mathrm{CDCl}_{3}\right) \delta: 167.35,166.13,165.97,164.80$, $137.96,137.53,137.41,137.20,134.60,133.54,133.35$, $133.15,129.85,129.78,129.72,129.55,129.04,128.99$, $128.69,128.62,128.56,128.51,128.48,128.43,128.40$, $128.32,128.24,128.09,127.73,127.67,125.32,101.19$, $98.51,97.34,82.24,80.26,78.09,77.90,75.66,75.59$, $75.31,74.75,73.63,70.06,68.73,67.82,63.44,63.17$, 62.66, 62.44, 55.40; HRMS (ESI) calcd for $\mathrm{C}_{75} \mathrm{H}_{72} \mathrm{~N}_{6} \mathrm{O}_{18} \mathrm{Na}$ : 1367.4795; Found: 1367.4803 .

Methyl(2-azido-3,4-O-benzyl-2-deoxy-6- $O$-benzoyl$\alpha$-D-glucopyranosyl)-( $\rightarrow 4$ )-(benzyl $2,3-O$-benzyl- $\beta$ - $D$ glucopyranosyluronate)-( $\rightarrow 4)-2$-azido-3-O-benzyl-2deoxy-6- $O$-benzoyl- $\alpha$ - $D$-glucopyranoside(6):

Procedure A: 给体 $\mathbf{9}(49 \mathrm{mg}, 0.1 \mathrm{mmol})$, 受体 12(172 mg, $0.2 \mathrm{mmol})$; 柱层析 $\left(V_{\mathrm{Tol}}: V_{\mathrm{EA}}=30: 1\right)$, 三糖 产物 6: $102 \mathrm{mg}, 76 \%, \alpha: \beta=6.3: 1.0$.

$[\alpha]_{\mathrm{D}}^{23}=58.0\left(c \quad 0.5, \mathrm{CHCl}_{3}\right) ;{ }^{1} \mathrm{H} \mathrm{NMR}(400 \mathrm{MHz}$, $\left.\mathrm{CDCl}_{3}\right) \delta: 8.03(\mathrm{~d}, 2 \mathrm{H}, J=7.2 \mathrm{~Hz}), 7.96(\mathrm{~d}, 2 \mathrm{H}, J=7.2 \mathrm{~Hz})$,
$7.53 \sim 7.20(\mathrm{~m}, 36 \mathrm{H}), 5.55(\mathrm{~d}, 1 \mathrm{H}, J=3.2 \mathrm{~Hz}), 5.51(\mathrm{t}, 1 \mathrm{H}$, $J=9.6 \mathrm{~Hz}), 5.35(\mathrm{t}, 1 \mathrm{H}, J=8.7 \mathrm{~Hz}), 5.05 \sim 5.03(\mathrm{~m}, 2 \mathrm{H})$, $5.00(\mathrm{~d}, 2 \mathrm{H}, J=1.5 \mathrm{~Hz}), 4.83 \sim 4.70(\mathrm{~m}, 5 \mathrm{H}), 4.64 \sim 4.50$ $(\mathrm{m}, 4 \mathrm{H}), 4.24 \sim 4.24(\mathrm{~m}, 3 \mathrm{H}), 4.15 \sim 3.98(\mathrm{~m}, 4 \mathrm{H}), 3.84 \sim$ $3.77(\mathrm{~m}, 5 \mathrm{H}), 3.72 \sim 3.66(\mathrm{~m}, 2 \mathrm{H}), 3.53(\mathrm{dd}, 1 \mathrm{H}, J=10.2$ $\mathrm{Hz}, 3.6 \mathrm{~Hz}), 3.45 \sim 3.41(\mathrm{~m}, 1 \mathrm{H}), 3.34 \sim 3.28(\mathrm{~m}, 5 \mathrm{H}) ;{ }^{13} \mathrm{C}$ NMR $\left(100 \mathrm{MHz}, \mathrm{CDCl}_{3}\right) \delta: 167.74,166.22,165.87$, $138.20,137.85,137.83,137.62,137.50,134.70,133.36$, $133.17,129.96,129.79,129.73,129.59,129.08,128.64$, $128.62,128.60,128.53,128.49,128.18,128.13,128.10$, $128.02,127.94,127.65,127.39,102.98,98.48,97.40$, $84.08,82.59,80.35,78.11,77.95,77.73,75.93,75.67$, $75.56,75.34,75.29,74.96,74.58,69.96,69.05,67.83$, $63.51,63.09,62.79,62.35,55.55$; HRMS (ESI) calcd for $\mathrm{C}_{75} \mathrm{H}_{74} \mathrm{~N}_{6} \mathrm{O}_{17} \mathrm{Na}: 1353.5003$; Found: 1353.5027.

Methyl(benzyl 2-O-benzoyl-3,4-O-benzyl- $\beta$ - $D$ glucopyranosyluronate)-( $\rightarrow 4)$-(2-azido-3-O-benzyl-2deoxy-6- $O$-benzoyl- $\alpha$ - $D$-glucopyranosyl)-( $\rightarrow 4)$-(benzyl 2-O-benzoyl-3- $O$-benzyl- $\beta$ - $D$-glucopyranosyluronate)$(1 \rightarrow 4)-2$-azido-3-O-benzyl-2-deoxy-6-O-benzoyl- $\alpha$ - $D$ glucopyranoside(7):

Procedure A: 给体 10(171 $\mathrm{mg}, 0.18 \mathrm{mmol})$, 受体 11(315 mg, $0.36 \mathrm{mmol})$; 柱层析 $\left(V_{\mathrm{Tol}}: V_{\mathrm{EA}}=25: 1\right)$, 四 糖产物 7: $237 \mathrm{mg}, 73 \%, \alpha: \beta=5.4: 1.0$.

$\left.[\alpha]_{\mathrm{D}}^{23}=68.2(c) 1.0, \mathrm{CHCl}_{3}\right) ;{ }^{1} \mathrm{H} \mathrm{NMR}(400 \mathrm{MHz}$, $\left.\mathrm{CDCl}_{3}\right) \delta: 8.06 \sim 7.99(\mathrm{~m}, 6 \mathrm{H}), 7.89(\mathrm{~d}, 2 \mathrm{H}, J=8.0 \mathrm{~Hz})$, $7.48 \sim 7.04(\mathrm{~m}, 48 \mathrm{H}), 5.45 \sim 5.37(\mathrm{~m}, 3 \mathrm{H}), 5.16(\mathrm{~d}, 1 \mathrm{H}$, $J=11.2 \mathrm{~Hz}), 4.99 \sim 4.97(\mathrm{~m}, 3 \mathrm{H}), 4.79(\mathrm{~d}, 1 \mathrm{H}, J=8.4 \mathrm{~Hz})$, $4.73 \sim 4.34(\mathrm{~m}, 14 \mathrm{H}), 4.37(\mathrm{dd}, 1 \mathrm{H}, J=12.0 \mathrm{~Hz}, 3.6 \mathrm{~Hz})$, $4.28(\mathrm{t}, 1 \mathrm{H}, J=9.6 \mathrm{~Hz}), 4.05 \sim 3.97(\mathrm{~m}, 2 \mathrm{H}), 3.93 \sim 3.83$ $(\mathrm{m}, 5 \mathrm{H}), 3.78 \sim 3.71(\mathrm{~m}, 3 \mathrm{H}), 3.66(\mathrm{~d}, 1 \mathrm{H}, J=10.4 \mathrm{~Hz})$, $3.28 \sim 3.25(\mathrm{~m}, 5 \mathrm{H}) ;{ }^{13} \mathrm{C} \mathrm{NMR}\left(100 \mathrm{MHz}, \mathrm{CDCl}_{3}\right) \delta$ : $167.49,167.09$, 165.97, 165.92, 164.86, 164.74, 138.06, $137.87,137.82,137.45,137.30,137.04,134.95,134.53$, $133.54,133.49,133.37,129.83,129.75,129.59,129.55$, $129.48,129.46,129.07,128.92,128.88,128.77,128.68$, $128.62,128.56,128.55,128.48,128.46,128.37,128.35$, $128.29,128.27,128.25,127.94,127.87,127.76,127.74$, $127.69,127.67,125.32,101.16,101.15,98.47,96.87$, $82.55,81.98,79.45,77.97,77.81,77.72,77.60,75.68$, $75.64,75.35,75.02,74.93,74.69,74.38,73.95,73.63$, $73.48,69.45,68.72,67.43,67.36,63.13,62.83,62.34$, 62.02, 55.38; ESI-MS $(\mathrm{m} / \mathrm{z}): 1828.4[\mathrm{M}+\mathrm{Na}]^{+}$.

Methyl(benzyl 2- $O$-benzoyl-3,4-O-benzyl- $\beta$ - $D$ glucopyranosyluronate)-( $\rightarrow 4)$-(2-azido-3-O-benzyl-2deoxy-6-O-benzoyl- $\alpha$ - $D$-glucopyranosyl)-(1 $\rightarrow 4)-($ benzyl $2,3-O$-benzyl- $\beta$ - $D$-glucopyranosyluronate)-( $\rightarrow 4)-2$ azido-3-O-benzyl-2-deoxy-6- $O$-benzoyl- $\alpha$ - $D$-glucopyran 
oside(8):

Procedure A: 给体 10(192 $\mathrm{mg}, 0.2 \mathrm{mmol})$, 受体 12(343 mg, $0.4 \mathrm{mmol})$; 柱层析 $\left(V_{\mathrm{Tol}}: V_{\mathrm{EA}}=30: 1\right)$, 四糖 产物 8: $236 \mathrm{mg}, 65 \%, \alpha: \beta=8.3: 1.0$.

${ }^{1} \mathrm{H}$ NMR (400 MHz, $\left.\mathrm{CDCl}_{3}\right) \delta: 7.98 \sim 7.95(\mathrm{~m}, 4 \mathrm{H})$, 7.89 (d, 2H, $J=8.4 \mathrm{~Hz}), 7.48 \sim 7.06(\mathrm{~m}, 49 \mathrm{H}), 5.50(\mathrm{~d}, 1 \mathrm{H}$, $J=4.0 \mathrm{~Hz}), 5.38(\mathrm{t}, 1 \mathrm{H}, J=8.8 \mathrm{~Hz}), 5.13(\mathrm{~d}, 1 \mathrm{H}, J=10.4$ $\mathrm{Hz}), 5.01 \sim 4.92(\mathrm{~m}, 4 \mathrm{H}), 4.79 \sim 4.73(\mathrm{~m}, 4 \mathrm{H}), 4.69 \sim 4.41$ $(\mathrm{m}, 13 \mathrm{H}), 4.34(\mathrm{dd}, 1 \mathrm{H}, J=12.4 \mathrm{~Hz}, 4.0 \mathrm{~Hz}), 4.11(\mathrm{t}, 1 \mathrm{H}$, $J=9.2 \mathrm{~Hz}), 4.03 \sim 3.90(\mathrm{~m}, 3 \mathrm{H}), 3.83 \sim 3.61(\mathrm{~m}, 8 \mathrm{H}), 3.46$ $(\mathrm{t}, 1 \mathrm{H}, J=8.0 \mathrm{~Hz}), 3.40(\mathrm{~s}, 2 \mathrm{H}), 3.31(\mathrm{dd}, 1 \mathrm{H}, J=10.4 \mathrm{~Hz}$, $3.6 \mathrm{~Hz}), 3.24$ (dd, $1 \mathrm{H}, J=10.4 \mathrm{~Hz}, 4.0 \mathrm{~Hz}) ;{ }^{13} \mathrm{C}$ NMR $(100$ $\left.\mathrm{MHz}, \mathrm{CDCl}_{3}\right) \delta: 167.59,167.49,166.08,165.86,164.87$, $138.13,138.06,137.82,137.77,137.53,137.38,135.05$, $134.62,133.56,133.46,133.43,129.93,129.69,129.68$, $129.64,129.54,129.26,129.03,128.99,128.73,128.70$, $128.69,128.67,128.64,128.59,128.55,128.52,128.49$, $128.37,128.07,128.05,128.00,127.94,127.86,127.76$, $127.70,127.54,102.95,101.22,98.44,96.89,84.20,82.68$, $82.11,79.55,78.07,77.91,77.70,77.62,77.36,75.97$, $75.81,75.61,75.48,75.40,75.17,75.01,74.29,74.08$, $73.52,69.37,69.07,67.48,63.05,62.88,62.20,62.13$, 55.60; ESI-MS $(\mathrm{m} / \mathrm{z}): 1813.0[\mathrm{M}+\mathrm{Na}]^{+}$.

肝素寡糖脱保护硫酸化的一般性步骤:

General Procedure B: 水解:

将化合物溶于四氢呋喃和甲醇的混合溶液 $\left(V_{\mathrm{THF}}\right.$ : $\left.V_{\mathrm{MeOH}}=1: 1,0.01 \mathrm{~mol} / \mathrm{L}\right)$, 慢慢加入 $3 \mathrm{~mol} / \mathrm{L} \mathrm{KOH}$ 水溶 液 $\left(V_{\mathrm{KOH}}: V_{\mathrm{THF}}=1: 1\right)$, 室温反应 $24 \mathrm{~h} . \operatorname{TLC}\left(V_{\mathrm{DCM}}\right.$ : $\left.V_{\mathrm{MeOH}}=10: 1\right)$ 检测反应完全后, 加入酸性树脂调至中 性. 使用旋转蒸发仪旋去反应液, 凝胶柱 $\mathrm{LH}-20\left(V_{\mathrm{DCM}}\right.$ : $\left.V_{\mathrm{MeOH}}=1: 1\right)$ 可分离出目标产物.

General Procedure C: $\boldsymbol{O}$-硫酸化:

将起始原料溶于 DMF 溶液 $(0.02 \mathrm{~mol} / \mathrm{L})$ 中, 加入三 氧化硫吡啶(10 equiv. per hydroxyl), 室温下反应 $4 \sim 8 \mathrm{~h}$. $\mathrm{TLC}$ (反相板, $V_{\mathrm{MeOH}}: V_{\mathrm{H} 2 \mathrm{O}}=3: 1$ )检测反应完全后, 可 以直接 $\mathrm{LH}-20\left(V_{\mathrm{DCM}}: V_{\mathrm{MeOH}}=1: 1\right)$ 提纯; 或者加入甲醇 搅拌 $15 \mathrm{~min}$, 使用旋转蒸发仪旋去反应液, 反相柱 $\mathrm{RP}-18$ 提纯, $\mathrm{MeOH}$ 和 $\mathrm{H}_{2} \mathrm{O}$ 作为洗脱剂, 梯度淋洗 $(0$ : $100 \rightarrow 75: 25, V: V$ 分离出目标分子, 之后通过 Dewex50WX4Na ${ }^{+}$柱交换成钠型.

General Procedure D: 叠氮还原以及 $\mathrm{N}$-硫酸化:

将起始原料溶于 $\mathrm{THF}(0.005 \mathrm{~mol} / \mathrm{L})$ 中, 加入 0.1 $\mathrm{mol} / \mathrm{L} \mathrm{NaOH}$ 水溶液 (5 mol per $-\mathrm{N}_{3}$ ), 之后滴加入 $\mathrm{PMe}_{3}$ 的 THF 溶液( 4 mol per $-\mathrm{N}_{3}$ ), 室温下反应 $8 \mathrm{~h}$. TLC(反 相板, $\left.V_{\mathrm{MeOH}}: V_{\mathrm{H} 2 \mathrm{O}}=2: 1\right)$ 检测反应完全后, 小心滴加 $0.1 \mathrm{~mol} / \mathrm{L} \mathrm{HCl}$ 溶液调 $\mathrm{pH}$ 至 7 8 , 使用旋转蒸发仪旋去 反应液.

残余物直接溶解在甲醇 $(0.005 \mathrm{~mol} / \mathrm{L})$ 中, 加入三乙 胺 $\left(V_{\mathrm{NEt} 3}: V_{\mathrm{MeOH}}=1: 3\right), 0.1 \mathrm{~mol} / \mathrm{L} \mathrm{NaOH}$ 水溶液 $(2 \mathrm{~mol}$ per $-\mathrm{NH}_{2}$ ), 充分搅拌后, 降温至 $0{ }^{\circ} \mathrm{C}$, 将三氧化硫吡 啶 (10 mol per - $\left.\mathrm{NH}_{2}\right)$ 加入, 冰浴下继续反应过夜. $\mathrm{TLC}$ (反相板, $V_{\mathrm{MeOH}}: V_{\mathrm{H} 2 \mathrm{O}}=1: 1$ )检测反应完全后, 浓 缩反应液, 反相柱 $\mathrm{RP}-18$ 提纯, $\mathrm{MeOH}$ 和 $\mathrm{H}_{2} \mathrm{O}$ 作为洗脱 剂, 梯度淋洗 $(0: 100 \rightarrow 50: 50, V: V)$ 分离出目标产物, 之后通过 Dewex50WX4 $\mathrm{Na}^{+}$柱交换成钠型.

General Procedure E: $\mathrm{Pd}(\mathrm{OH})_{2} / \mathrm{C}$ 脱保护:

将起始原料溶于甲醇和水的混合溶液 $\left(V_{\mathrm{MeOH}}\right.$ : $\left.V_{\mathrm{H} 2 \mathrm{O}}=3: 1\right)$ 中, 室温 $1013 \mathrm{kPa} \mathrm{H}_{2}$ 下氢化 $3 \mathrm{~d}$, 使用滤纸 过滤掉 $\mathrm{Pd}(\mathrm{OH})_{2} / \mathrm{C}$, 旋干得到目标分子. 如果产物中残 余少量 $\mathrm{Pd}(\mathrm{OH})_{2} / \mathrm{C}$, 可使用凝胶柱 $\mathrm{G}-10$ 提纯.

Methyl(3,4-O-benzyl- $\beta$ - $D$-glucopyranosyluronate)$(1 \rightarrow 4)$-(2-azido-3-O-benzyl-2-deoxy- $\alpha$ - $D$-glucopyranosy l)-(1 $\rightarrow 4)$-(3-O-benzyl- $\beta$ - $D$-glucopyranosyluronate)-( $\rightarrow$ 4)-2-azido-3- $O$-benzyl-2-deoxy- $\alpha$ - $D$-glucopyranoside(13 ):

Procedure B: 起始原料 $7 \propto(134 \mathrm{mg}, 0.074 \mathrm{mmol})$; 产物 13(85 mg, 96\%).

$[\alpha]_{\mathrm{D}}^{24}=46.2\left(c \quad 1.0, \mathrm{CHCl}_{3}\right) ;{ }^{1} \mathrm{H}$ NMR $(400 \mathrm{MHz}$, $\left.\mathrm{CD}_{3} \mathrm{OD}\right) \delta: 7.47 \sim 7.21(\mathrm{~m}, 25 \mathrm{H}), 5.52(\mathrm{~d}, 1 \mathrm{H}, J=2.8 \mathrm{~Hz})$, $5.13 \sim 5.07(\mathrm{~m}, 3 \mathrm{H}), 4.91 \sim 4.63(\mathrm{~m}, 10 \mathrm{H}), 4.06 \sim 3.48(\mathrm{~m}$, $18 \mathrm{H}), 3.31(\mathrm{~s}, 3 \mathrm{H}), 3.20(\mathrm{dd}, 1 \mathrm{H}, J=9.6 \mathrm{~Hz}, 2.8 \mathrm{~Hz})$, $3.10 \sim 3.08(\mathrm{~m}, 1 \mathrm{H}) ;{ }^{13} \mathrm{C}$ NMR $\left(100 \mathrm{MHz}, \mathrm{CD}_{3} \mathrm{OD}\right) \delta$ : $140.19,140.06,139.76,139.71,139.56,130.01,129.98$, $129.39,129.36,129.31,129.09,129.04,129.01,128.77$, $128.73,128.71,128.67,128.64,104.69,104.56,100.11$, $99.19,86.13,85.48,80.95,79.55,79.21,78.74,77.87$, $76.74,76.40,76.36,76.14,75.99,73.14,72.77,64.29$, 64.16, 61.06, 60.56, 55.62; HRMS (ESI) calcd for $\mathrm{C}_{60} \mathrm{H}_{68} \mathrm{~N}_{6} \mathrm{O}_{21} \mathrm{Na}$ : 1231.4330; Found: 1231.4300 .

Methyl(3,4-O-benzyl-2- $O$-sulfo- $\beta$ - $D$-glucopyranosy luronate)-( $\rightarrow 4)$-(2-azido-3- $O$-benzyl-6- $O$-sulfo-2-deoxy - $\alpha$ - $D$-glucopyranosyl)-( $\rightarrow 4)-(3-O$-benzyl-2- $O$-sulfo- $\beta$ - $D$ -glucopyanosyluronate)-(1 $\rightarrow 4)-2$-azido-3-O-benzyl-6-Osulfo-2-deoxy- $\alpha$ - $D$-glucopyranoside(14):

Procedure C: 起始原料 $13(22 \mathrm{mg}, 0.018 \mathrm{mmol})$; 产 物 14(28 mg, 99\%).

${ }^{1} \mathrm{H}$ NMR (400 MHz, $\left.\mathrm{CD}_{3} \mathrm{OD}\right) \delta: 7.55 \sim 7.23(\mathrm{~m}, 25 \mathrm{H})$, $5.49(\mathrm{~d}, 1 \mathrm{H}, J=3.6 \mathrm{~Hz}), 5.37(\mathrm{~d}, 1 \mathrm{H}, J=9.6 \mathrm{~Hz}), 5.27 \sim$ $5.18(\mathrm{~m}, 3 \mathrm{H}), 5.07 \sim 5.02(\mathrm{~m}, 2 \mathrm{H}), 4.79 \sim 4.67(\mathrm{~m}, 9 \mathrm{H})$, $4.50(\mathrm{~m}, 2 \mathrm{H}), 4.32 \sim 4.23(\mathrm{~m}, 2 \mathrm{H}), 4.07 \sim 3.87(\mathrm{~m}, 12 \mathrm{H})$, $3.41(\mathrm{~s}, 3 \mathrm{H}), 3.26 \sim 3.20(\mathrm{~m}, 2 \mathrm{H})$; ESI-MS $(\mathrm{m} / \mathrm{z}): 509.1$ $[\mathrm{M}+3 \mathrm{H}]^{3-}$.

Methyl(3,4-O-benzyl-2- $O$-sulfo- $\beta$ - $D$-glucopyranosyluronate)-( $1 \rightarrow 4)-(2-N$-sulfo-3-O-benzyl-6- $O$-sulfo-2deoxy- $\alpha$ - $D$-glucopyranosyl)-( $\rightarrow 4)-(3-O$-benzyl-2- $O$ sulfo- $\beta$ - $D$-glucopyanosyluronate)-( $\rightarrow 4)-2-N$-sulfo-3-O benzyl-6- $O$-sulfo-2-deoxy- $\alpha$ - $D$-glucopyranoside(15):

Procedure D: 起始原料 14(28 mg, $0.018 \mathrm{mmol})$; 产 
物 $\mathbf{1 5}$ (25 mg, 86\%).

${ }^{1} \mathrm{H}$ NMR (400 MHz, $\left.\mathrm{CD}_{3} \mathrm{OD}\right) \delta: 7.63 \sim 7.14(\mathrm{~m}, 25 \mathrm{H})$, $5.46(\mathrm{~d}, 1 \mathrm{H}), 5.12 \sim 4.98(\mathrm{~m}, 5 \mathrm{H}), 4.94 \sim 4.83(\mathrm{~m}, 3 \mathrm{H})$, $4.72 \sim 4.59(\mathrm{~m}, 7 \mathrm{H}), 4.51 \sim 4.47(\mathrm{~m}, 2 \mathrm{H}), 4.31 \sim 4.19(\mathrm{~m}$, $5 \mathrm{H}), 4.09 \sim 3.95(\mathrm{~m}, 6 \mathrm{H}), 3.89 \sim 3.74(\mathrm{~m}, 3 \mathrm{H}), 3.67 \sim 3.66$ $(\mathrm{m}, 1 \mathrm{H}), 3.50 \sim 3.41(\mathrm{~m}, 4 \mathrm{H})$; ESI-MS $(\mathrm{m} / \mathrm{z}): 545.1$ $[\mathrm{M}+5 \mathrm{H}]^{3-}$.

Methyl(2- $O$-sulfo- $\beta$ - $D$-glucopyranosyluronate)$(1 \rightarrow 4)-(2-N$-sulfo-6- $O$-sulfo-2-deoxy- $\alpha$ - $D$-glucopyranosyl)-( $1 \rightarrow 4)-(2-O$-sulfo- $\beta$ - $D$-glucopyanosyluronate)-( $\rightarrow$ 4)-2- $N$-sulfo-6- $O$-sulfo-2-deoxy- $\alpha$ - $D$-glucopyranoside(3):

Procedure E: 起始原料 15(25 mg, $0.016 \mathrm{mmol})$; 产 物 3(19 mg, 99\%).

${ }^{1} \mathrm{H}$ NMR (400 MHz, $\left.\mathrm{D}_{2} \mathrm{O}\right) \delta: 5.51(\mathrm{~d}, 1 \mathrm{H}, J=3.6 \mathrm{~Hz})$, $5.03(\mathrm{~d}, 1 \mathrm{H}, J=3.2 \mathrm{~Hz}), 4.79(\mathrm{~d}, 1 \mathrm{H}), 4.59 \sim 4.54(\mathrm{~m}, 2 \mathrm{H})$, $4.27 \sim 4.09(\mathrm{~m}, 4 \mathrm{H}), 4.04 \sim 3.95(\mathrm{~m}, 3 \mathrm{H}), 3.85 \sim 3.69(\mathrm{~m}$, $9 \mathrm{H}), 3.59(\mathrm{t}, 1 \mathrm{H}, J=9.6 \mathrm{~Hz}), 3.42(\mathrm{~s}, 3 \mathrm{H}), 3.31 \sim 3.28(\mathrm{~m}$, $2 \mathrm{H}) ;{ }^{13} \mathrm{C}$ NMR $\left(100 \mathrm{MHz}, \mathrm{D}_{2} \mathrm{O}\right) \delta: 100.06,99.82,98.58$, 98.23, 79.78, 79.68, 78.79, 78.06, 77.58, 76.55, 75.66, $74.53,74.46,71.71,69.43,69.09,68.04,65.93,65.83$, 57.86, 57.19, 55.48, 55.44; ESI-MS $(\mathrm{m} / \mathrm{z}): 394.8[\mathrm{M}+$ $5 \mathrm{H}]^{3-}$

Methyl(2- $N$-sulfo-6- $O$-sulfo-2-deoxy- $\alpha$ - $D$-glucopyranosyl)-(1 $\rightarrow 4)-(2-O$-sulfo- $\beta$ - $D$-glucopyanosyluronate)-( $1 \rightarrow 4)-2-N$-sulfo-6-O-sulfo-2-deoxy- $\alpha$ - $D$-glucopyranoside(1):

Procedure E: 起始原料 $5(67 \mathrm{mg}, 0.05 \mathrm{mmol})$; 产物 1(25 mg, 54\%).

${ }^{1} \mathrm{H}$ NMR (400 MHz, D $\left.2 \mathrm{O}\right) \delta: 5.69(\mathrm{~d}, 1 \mathrm{H}, J=2.4 \mathrm{~Hz})$, 5.07 (d, 1H, $J=2.8 \mathrm{~Hz}), 4.79(\mathrm{~d}, 1 \mathrm{H}), 4.62$ (d, 1H, $J=10.0$ $\mathrm{Hz}), 4.40$ (d, 1H, $J=10.4 \mathrm{~Hz}), 4.29$ (d, 1H, $J=11.2 \mathrm{~Hz})$, $4.23 \sim 4.15(\mathrm{~m}, 2 \mathrm{H}), 4.04 \sim 3.60(\mathrm{~m}, 9 \mathrm{H}), 3.46(\mathrm{~s}, 3 \mathrm{H})$, $3.33 \sim 3.27(\mathrm{~m}, 2 \mathrm{H}) ;{ }^{13} \mathrm{C}$ NMR $\left(100 \mathrm{MHz}, \mathrm{D}_{2} \mathrm{O}\right) \delta: 100.07$, $98.31,97.82,79.93,77.91,76.47,75.05,71.30,69.95$, $69.48,69.08,68.10,66.39,65.97,57.94,57.26,55.51$; ESI-MS $(\mathrm{m} / \mathrm{z}): 309.2[\mathrm{M}+3 \mathrm{H}]^{3-}, 464.2[\mathrm{M}+4 \mathrm{H}]^{2-}$.

Methyl(2- $N$-sulfo-6- $O$-sulfo-2-deoxy- $\alpha$ - $D$-glucopyranosyl)-( $1 \rightarrow 4)-\beta$ - $D$-glucopyanosyluronate-( $1 \rightarrow 4)-2$ $\boldsymbol{N}$-sulfo-6- $\boldsymbol{O}$-sulfo-2-deoxy- $\boldsymbol{\alpha}-\boldsymbol{D}$-glucopyranoside(2):

Procedure B, C, D, E: 起始原料 6(75 mg, 0.056 $\mathrm{mmol})$; 产物 2(34 mg, 71\%).

${ }^{1} \mathrm{H}$ NMR (400 MHz, $\left.\mathrm{D}_{2} \mathrm{O}\right) \delta: 5.67$ (d, 2H, $\left.J=3.6 \mathrm{~Hz}\right)$, $5.07(\mathrm{~d}, 1 \mathrm{H}, J=3.6 \mathrm{~Hz}), 4.63(\mathrm{~d}, 1 \mathrm{H}, J=8.0 \mathrm{~Hz}), 4.43 \sim$ $4.35(\mathrm{~m}, 3 \mathrm{H}), 4.19(\mathrm{~d}, 1 \mathrm{H}, J=10.8 \mathrm{~Hz}), 4.03 \sim 4.01(\mathrm{~m}$, $1 \mathrm{H}), 3.92 \sim 3.80(\mathrm{~m}, 4 \mathrm{H}), 3.77 \sim 3.70(\mathrm{~m}, 2 \mathrm{H}), 3.67 \sim 3.58$ $(\mathrm{m}, 2 \mathrm{H}), 3.46(\mathrm{~s}, 3 \mathrm{H}), 3.41(\mathrm{t}, 1 \mathrm{H}, J=8.4 \mathrm{~Hz}), 3.34 \sim 3.28$ $(\mathrm{m}, 2 \mathrm{H}) ;{ }^{13} \mathrm{C}$ NMR $\left(100 \mathrm{MHz}, \mathrm{D}_{2} \mathrm{O}\right) \delta: 102.14,98.24$, 97.40, 78.53, 76.43, 76.28, 76.17, 72.99, 71.12, 69.79,
$69.67,69.04,69.23,66.52,66.35,57.93,57.25,55.52$; ESI-MS $(\mathrm{m} / \mathrm{z}): 282.3[\mathrm{M}+2 \mathrm{H}]^{3-} ; 424.2[\mathrm{M}+3 \mathrm{H}]^{2-}$.

Methyl(2- $O$-sulfo- $\beta$ - $D$-glucopyranosyluronate)$(1 \rightarrow 4)-(2-N$-sulfo-6- $O$-sulfo-2-deoxy- $\alpha$ - $D$-glucopyranosyl)-( $1 \rightarrow 4)-\beta$ - $D$-glucopyanosyluronate-( $\rightarrow 4)-2-N$-sulfo6- $O$-sulfo-2-deoxy- $\alpha$ - $D$-glucopyranoside(4):

Procedure B, C, D, E: 起始原料 8(30 mg, 0.017 $\mathrm{mmol})$; 产物 $\mathbf{4}(10 \mathrm{mg}, 52 \%)$.

${ }^{1} \mathrm{H}$ NMR (400 MHz, $\left.\mathrm{D}_{2} \mathrm{O}\right) \delta: 5.52(\mathrm{~d}, 1 \mathrm{H}, J=3.6 \mathrm{~Hz})$, $5.05(\mathrm{~d}, 1 \mathrm{H}, J=3.2 \mathrm{~Hz}), 4.79$ (d, 1H), 4.60 (d, 1H, $J=8.0$ $\mathrm{Hz}), 4.56(\mathrm{~d}, 1 \mathrm{H}, J=10.8 \mathrm{~Hz}), 4.42(\mathrm{~d}, 1 \mathrm{H}, J=10.8 \mathrm{~Hz})$, $4.33(\mathrm{dd}, 1 \mathrm{H}, J=11.2 \mathrm{~Hz}, 3.6 \mathrm{~Hz}), 4.20(\mathrm{~d}, 1 \mathrm{H}, J=10.8$ $\mathrm{Hz}), 4.12(\mathrm{t}, 1 \mathrm{H}, J=8.8 \mathrm{~Hz}), 4.03 \sim 4.01(\mathrm{~m}, 2 \mathrm{H}), 3.88 \sim$ $3.68(\mathrm{~m}, 9 \mathrm{H}), 3.60(\mathrm{t}, 1 \mathrm{H}, J=9.6 \mathrm{~Hz}), 3.44(\mathrm{~s}, 3 \mathrm{H}), 3.42 \sim$ $3.31(\mathrm{~m}, 3 \mathrm{H}) ;{ }^{13} \mathrm{C} \mathrm{NMR}\left(100 \mathrm{MHz}, \mathrm{D}_{2} \mathrm{O}\right) \delta: 181.53,175.60$, 102.23, 100.81, 99.84, 98.21, 79.70, 78.81, 78.67, 77.58, $76.63,75.74,75.68,74.48,72.96,71.74,69.70,69.36$, 69.01, 68.25, 66.62, 65.87, 57.86, 57.24, 55.50; ESI-MS $(\mathrm{m} / \mathrm{z}): 367.5[\mathrm{M}+4 \mathrm{H}]^{3-}$.

\section{References}

[1] Molean, J. Am. J. Physiol. 1916, 41, 250.

[2] Capila, R. J. Linhardt, D. Angew. Chem. Int. Ed. 2002, 41, 390.

[3] (a) Petitou, M.; van Boeckel, C. A. A. Angew. Chem. Int. Ed. Engl. 1993, 32, 1671; (b) Petitou, M.; van Boeckel, C. A. A. Angew. Chem. Int. Ed. 2004, 43, 3118.

[4] (a) Casu, B. Adv. Carbohydr. Chem. Biochem. 1985, 43, 51; (b) Gao, N. G.; Cheng, X. L.; Yang, J.; Zhang, S. Z. Prog. Biotechnol. 1999, 19(5), 4 (in Chinese). (高宁国，程秀兰，杨敬，张树政，生物工程 进展, 1999, 19(5), 4.)

[5] Poletti, L.; Lay, L. Eur. J. Org. Chem. 2003, 2999; (b) Karst, N. A.; Linhardt, R. J. Curr. Med. Chem. 2003, 10, 1993.

[6] Vlodavsky, I.; Goldshmidt, O.; Zcharia, E.; Metzger, S.; Chajek-Shaul, T.; Atzmon, R.; Guatta-Rangini, Z.; Friedmann, Y. Biochimie 2001, 83, 831.

[7] Elkin, M.; Ilan, N.; Ishai-Michaeli, R. FASEB 2001, 15, 1661.

[8] Bartleet, M. R.; Underwood, P. A.; Parish, C. R. Immunol. Cell Biol. 1995, 73, 113.

[9] Bingley, J. A.; Hayward, I. P.; Campbell, J. H. J. Vasc. Surg. 1998 $28,308$.

[10] Okada, Y.; Yamada, S.; Toyoshima, M.; Dong, J.; Nakajima, M.; Sugahara, K. J. Biol. Chem. 2002, 277, 42488.

[11] For reviews, see: (a) Gin, D. J. Carbohydr. Chem. 2002, 21, 645; (b) O’Neill, S.; Rodriguez, J.; Walczak, M. A. Chem. Asian J. 2018, 13, 2978; (c) Ryan, D. A.; Gin, D. Y. Glycoside Synthesis from 1-Oxygen Substituted Glycosyl Donors. In Handbook of Chemical Glycosylation, Ed.: Demchenko, A. V., Wiley-Ver \& Co. KGaA, Weinheim, 2008, pp. 95-143.

[12] (a) Chen, J.; Zhou, Y.; Chen, C.; Xu, W.; Yu, B. Carbohydr. Res. 2008, 343, 2853; (b) Xu, P.; Xu, W.; Dai, Y.; Yang, Y.; Yu, B. Org. Chem. Front. 2014, 1, 405.

[13] Jiang, L.; Chan, T. Tetrahedron Lett. 1998, 39, 355.

[14] Epp, J. B.; Widlanski, T. S. J. Org. Chem., 1999, 64, 293.

[15] (a) Yin, X.; Yan, J.; Ji, S.; Wang, F.; Cao, H. Chin. J. Org. Chem. 2012, 32, 1388; (b) Li, J.; Dai, Y.; Li, W.; Laval, S.; Xu, P.; Yu, B. Asian J. Org. Chem. 2015, 4, 756; (c) Mende, M.; Bednarek, C.; Wawryszyn, M.; Sauter, P.; Biskup, M. B.; Schepers, U.; Brase, S. Chem. Rev. 2016, 116, 8193

[16] (a) Nishida, Y.; Shingu, Y.; Dohi, H.; Kobayashi, K. Org. Lett. 2003, 5, 2377; (b) Kim, K. S.; Fulse, D. B.; Baek, J. Y.; Lee, B.-Y.; Jeon, H. B. J. Am. Chem. Soc. 2008, 130, 8537; (c) Mossotti, M.; Panza, L. J. Org. Chem. 2011, 76, 9122; (d) Nogueira, J. M.; Nguyen, S. H.; Bennett, C. S. Org. Lett. 2011, 13, 2814; (e) Nogueira, J. M.; 
Bylsma, M.; Bright, D. K.; Bennett, C. S. Angew. Chem. Int. Ed. 2016, 55, 10088; (f) Zhou, M.-H.; Wilbur, D. J.; Kwan, E. E.; Bennett, C. S. J. Am. Chem. Soc. 2019, 141, 16743; (g) Dyapa, R.; Dockery, L. T.; Walczak, M. A. Org. Biomol. Chem. 2017, 15, 51; (h) Ghosh, T.; Mukherji, A.; Srivastava, H. K.; Kancharla, P. K. Org. Biomol. Chem. 2018, 16, 2870; (i) Manhas, S.; Taylor, M. S. Carbohydr. Res. 2018, 470, 42; (j) Cai, L.; Zeng, J.; Li, T.; Xiao, Y.; Ma, X.; Xiao, X.; Zhang, Q.; Meng, L.; Wan, Q. Chin. J. Chem. 2020, $38,43$.

[17] (a) Garcia, B. A.; Poole, J. L.; Gin, D. Y. J. Am. Chem. Soc. 1997, 119, 7597; (b) Garcia, B. A.; Gin, D. Y. J. Am. Chem. Soc. 2000, 122,4269 .

[18] (a) Codée, J. D. C.; van den Bos, L. J.; Litjens, R. E. J. N.; Overkleeft, H. S.; van Boom J. H.; van der Marel, G. A. Org. Lett. 2003, 5 , 1947; (b) van den Bos, L. J.; Codée, J. D. C.; van Boom J. H.; Overkleeft, H. S.; van der Marel, G. A. Org. Biomol. Chem. 2003, 1, 4160; (c) van den Bos, L. J.; Codée, J. D. C.; van Boom, J. H.; van der Toorn, J. C.; Boltje, T. J.; van Boom J. H.; Overkleeft, H. S.; van der Marel, G. A. Org. Lett. 2004, 6, 2165; (d) Codée, J. D. C.; Stubba, B.; Schiattarella, M.; Overkleeft, H. S.; van Boeckel, C. A. A.; van Boom J. H.; van der Marel, G. A. J. Am. Chem. Soc. 2005, 127, 3767.

[19] Zhou, Y. Ph.D. Dissertation, Shanghai Institute of Organic Chemistry, CAS, Shanghai, 2005 (in Chinese). (周映, 博士论文, 中国科学院上海有机化学研究所, 上海, 2005.)

[20] (a) Arungundram, S.; Al-Mafraji, K.; Asong, J.; Leach III, F. E.; Amster, J., Venot, A.; Turnbull, J. E.; and Boons, G. J. Am. Chem. Soc. 2009, 131, 17394; (b) Hu, Y.; Lin, S.; Huang, C.; Zulueta, M. M. L.; Liu J.; Chang, W.; Hung, S.-C. Nat. Chem. 2011, 3, 557; (c) Zulueta, M.; Lin, S.; Lin, Y.; Huang, C.; Wang, C.; Ku, C.; Shi, Z.; Wong, C.-H.; Hung, S.-C. J. Am. Chem. Soc. 2012, 134, 8988; (d) Wang, Z.; Xu, Y.; Yang B., Tiruchinapally, G.; Sun, B.; Liu, R.; Dulaney, S.; Liu, J.; Huang, X. Chem. Eur. J. 2010, 16, 8365; (e) Haller, M.; Boons, G-J. J. Chem. Soc., Perkin Trans.1. 2001, 814; (f) Lubineau, A.; Lortat-Jacob, H.; Gavard, O.; Sarrazin, S.; Bonnaffé, D. Chem. Eur. J. 2004, 10, 4265; (g) Lin, F.; Lian, G.; Zhou, Y. Carbohydr. Res. 2013, 371, 32; (h) Li, T.; Ye, H.; Cao, X.; Wang, J.;
Liu, Y.; Zhou, L.; Liu, Q.; Wang, W.; Shen, J. Zhao, W.; Wang, P. ChemMedChem 2014, 9, 1071; (i) Xu, P.; Laval, S.; Guo, Z.; Yu, B. Org. Chem. Front. 2016, 3, 103; (j) Dai, X.; Liu, W.; Zhou, Q.; Cheng, C.; Yang, C.; Wang, S.; Zhang, M.; Tang, P.; Song, H.; Zhang, D.; Qin, Y. J. Org. Chem. 2016, 81, 162; (k) Ding, Y.; Vara Prasad C. V. N. S.; Bai, H.; Wang, B. Bioorg. Med. Chem. Lett. 2017, 27, 2424; (1) Jin, H.; Chen, Q.; Zhang, Y.; Hao, K. Zhang, G.; Zhao, W. Org. Chem. Front. 2019, 6, 3116.

[21] (a) Orgueira, H. A.; Bartolozzi, A.; Schell, P.; Litjens, R. E. J. N.; Palmacci, E. R.; Seeberger, P. H. Chem. Eur. J. 2003, 9, 140; (b) Noti, C.; de Paz, J. L.; Polito, L.; Seeberger, P. H. Chem. Eur. J. 2006, 12, 8664; (c) Zhang, L.; Xu, P.; Liu, B.; Yu, B. J. Org. Chem. 2020, DOI: 10.1021/acs.joc.0c01009.

[22] (a) Mungall, W. S.; Greene, G. L.; Heavner, G. A.; Letsinger, R. L. J. Org. Chem. 1975, 40, 1659; (b) Alper, P. B.; Hendrix, M.; Sears, P.; Wong, C. H. J. Am. Chem. Soc. 1998, 120, 1965.

[23] Bayley, H.; Standring, D. N.; Knowles, J. R. Tetrahedron Lett. 1978, $19,3633$.

[24] Corey, E. J.; Nicolaou, K. C.; Balanson, R. D.; Machida, Y. Synthesis 1975, 590 .

[25] Lee, J.-C.; Lu, X.-A.; Kulkarni, S. S.; Wen, Y.-S.; Hung, S.-C. J. Am. Chem. Soc. 2003, 126, 476.

[26] (a) Benati, L.; Montevecchi, P. C.; Nanni, D.; Spagnolo, P.; Volta, M. Tetrahedron Lett. 1995, 36, 7313; (b) Goulaouic-Dubois, C.; Hesse, M. Tetrahedron Lett. 1995, 36, 7427.

[27] Brewer, M.; Rich, D. H. Org. Lett. 2001, 3, 945.

[28] Gregory, J.; Lohman, S.; Seeberger, P. H. J .Org. Chem. 2004, 69, 4081.

[29] Yang, B.; Yoshida, K.; Yin, Z.; Dai, H.; Kavunja, H.; El-Dakdouki, M. H.; Sungsuwan, S.; Dulaney, S. B.; Huang, X. Angew. Chem., Int. Ed. 2012, 51, 10185.

[30] (a) Lee, J.-C.; Lu, X.-A.; Kulkarni, S. S.; Wen, Y.-S.; Hung, S.-C. J. Am. Chem. Soc. 2003, 126, 476; (b) Lee, J. C. Ph.D. Dissertation, Tsing Hua University, Taiwan, 2005 (in Chinese). (李静琪, 博士论 文, 台湾清华大学, 台湾, 2003.)

[31] Dilhas, A.; Lucas, R.; Loureiro-Morais, L.; Hersant, Y.; Bonnaffé, D. J. Comb. Chem. 2008, 10, 166.

(Yang, X.) 